\title{
Pediatric Neuromyelitis Optica Spectrum Disorder: Case Series and Literature Review
}

\author{
Michela Ada Noris Ferilli ${ }^{1}{ }^{*}$, Roberto Paparella ${ }^{2}\left(\right.$, Ilaria Morandini ${ }^{3}$, Laura Papetti ${ }^{1}$, Lorenzo Figà Talamanca ${ }^{4}$, \\ Claudia Ruscitto ${ }^{3}$, Fabiana Ursitti ${ }^{1}$, Romina Moavero ${ }^{1,3} \mathbb{0}$, Giorgia Sforza ${ }^{1}$, Samuela Tarantino ${ }^{5}$, \\ Martina Proietti Checchi ${ }^{5}$, Federico Vigevano ${ }^{1}$ and Massimiliano Valeriani ${ }^{1,6}{ }_{\mathbb{D}}$ \\ 1 Neuroscience Department, Bambino Gesù Children's Hospital IRCCS, 00165 Rome, Italy; \\ laura.papetti@opbg.net (L.P.); fabiana.ursitti@opbg.net (F.U.); romina.moavero@opbg.net (R.M.); \\ giorgia.sforza@opbg.net (G.S.); federico.vigevano@opbg.net (F.V.); massimiliano.valeriani@opbg.net (M.V.) \\ 2 Department of Maternal and Child Health and Urology, Sapienza University of Rome, 00165 Rome, Italy; \\ roberto.paparella@uniroma1.it \\ 3 Child Neurology Unit, Systems Medicine Department, Tor Vergata University Hospital of Rome, \\ 00165 Rome, Italy; ilaria.morandini@ptvonline.it (I.M.); claudia.ruscitto@ptvonline.it (C.R.) \\ 4 Imaging Department, Bambino Gesù Children's Hospital IRCCS, 00165 Rome, Italy; \\ lorenzo.figatalamanca@opbg.net \\ 5 Unit of Clinical Psychology, Neuroscience Department, Bambino Gesù Children's Hospital IRCCS, \\ 00165 Rome, Italy; samuela.tarantino@opbg.net (S.T.); martina.proietti@opbg.net (M.P.C.) \\ 6 Center for Sensory-Motor Interaction, Denmark Neurology Unit, Aalborg University, 9220 Aalborg, Denmark \\ * Correspondence: michela.ferilli@opbg.net
}

Citation: Ferilli, M.A.N.; Paparella, R.; Morandini, I.; Papetti, L.; Figà Talamanca, L.; Ruscitto, C.; Ursitti, F.; Moavero, R.; Sforza, G.; Tarantino, S.; et al. Pediatric Neuromyelitis Optica Spectrum Disorder: Case Series and Literature Review. Life 2022, 12, 19. https://doi.org/10.3390/life12010019

Academic Editors: Hueng-Chuen Fan, Chuan-Mu Chen, Yih-Jing Lee,

Ching-Shiang Chi and Shyi-Jou Chen

Received: 9 November 2021

Accepted: 21 December 2021

Published: 23 December 2021

Publisher's Note: MDPI stays neutral with regard to jurisdictional claims in published maps and institutional affiliations.

Copyright: (c) 2021 by the authors. Licensee MDPI, Basel, Switzerland. This article is an open access article distributed under the terms and conditions of the Creative Commons Attribution (CC BY) license (https:// creativecommons.org/licenses/by/ $4.0 /)$.

\begin{abstract}
Neuromyelitis Optica Spectrum Disorder (NMOSD) is a central nervous system (CNS) inflammatory demyelinating disease characterized by recurrent inflammatory events that primarily involve optic nerves and the spinal cord, but also affect other regions of the CNS, including hypothalamus, area postrema and periaqueductal gray matter. The aquaporin-4 antibody (AQP4-IgG) is specific for NMOSD. Recently, myelin oligodendrocyte glycoprotein antibodies (MOG-IgG) have been found in a group of AQP4-IgG negative patients. NMOSD is rare among children and adolescents, but early diagnosis is important to start adequate therapy. In this report, we present cases of seven pediatric patients with NMOSD and we review the clinical and neuroimaging characteristics, diagnosis, and treatment of NMOSD in children.
\end{abstract}

Keywords: neuromyelitis optica spectrum disorder; aquaporin-4 antibody; myelin oligodendrocyte glycoprotein antibodies; optic neuritis; longitudinally extensive transverse myelitis; children

\section{Introduction}

Neuromyelitis Optica Spectrum Disorder (NMOSD) is a rare central nervous system (CNS) inflammatory demyelinating disease characterized by recurrent inflammatory events, primarily involving optic nerves and the spinal cord, but also affecting other regions of the CNS, including hypothalamus, area postrema and periaqueductal gray matter.

It is stratified by the aquaporin-4 antibody (AQPA-IgG) into AQP4-IgG-positive NMOSD and seronegative NMOSD. AQP4-IgG is a sensitive and specific marker of NMOSD and has a function in its immunopathogenesis.

AQP4 is a water channel protein expressed on astrocyte foot processes at the bloodbrain barrier, especially in the periaqueductal and periventricular regions and at the level of the spinal cord gray matter [1].

AQP4-IgG positive NMOSD could be classified as an autoimmune astrocytopathic disease, in which the AQP4-IgG is thought to cause the internalization and degradation of AQP4, dysregulation of water and glutamate homeostasis, activation of the classical complement pathway, and antibody-mediated cytotoxicity.

Recent studies have shown that neutrophils, macrophages, and natural killer (NK) cells contribute to the formation of lesions in NMOSD through different mechanisms, 
such as the production of elastase, the secretion of cytokines, phagocytosis, and antibodydependent cytotoxicity [2]. Eosinophils are also involved in the pathogenesis of lesions in NMOSD, and these cells have been shown to contribute to tissue damage [3].

AQP4-IgG seronegativity in 10-25\% of NMOSD patients suggests that there are other mechanisms involved in NMOSD pathogenesis [4]. More recently, myelin oligodendrocyte glycoprotein antibodies (MOG-IgG) have been found in a group of AQP4-IgG negative patients, which have a different pathogenesis, younger age at presentation, fewer relapses, and a better outcome [5-7].

Approximately $3-5 \%$ of NMOSD cases are reported to be pediatric onset, before 18 years of age [8-11]. Early differentiation of NMOSD from other acquired demyelinating syndromes (ADS) of the CNS, such as acute disseminated encephalomyelitis (ADEM) and multiple sclerosis (MS), is critical for starting opportune treatment. In fact, it is now known that some disease-modifying therapies used for MS, including interferons, natalizumab and fingolimod, are ineffective in patients with NMOSD and may precipitate the course of the disease [12-14].

In this article we present cases of seven pediatric patients with NMOSD and we review the literature related to clinical and neuroimaging characteristics, diagnosis and treatment of pediatric NMOSD.

\section{Clinical Cases}

Clinical, radiological, and laboratory findings of our patients are reported in Table 1.

Table 1. Clinical, radiological and laboratory findings of our patients with NMOSD.

\begin{tabular}{|c|c|c|c|c|c|c|c|c|c|c|c|c|}
\hline $\mathrm{Pt}$ & $\begin{array}{c}\text { Gen, } \\
\text { Age (y) }\end{array}$ & Symptoms/Signs & $\begin{array}{c}\text { MRI } \\
\text { T2-Hyperintense } \\
\text { Lesions } \\
\end{array}$ & $\begin{array}{c}\text { Gd En- } \\
\text { hancement }\end{array}$ & $\begin{array}{l}\text { Antibody } \\
\text { Status }\end{array}$ & CSF OCBs & $\begin{array}{c}\text { Evoked } \\
\text { Potentials }\end{array}$ & $\begin{array}{c}\text { EDSS at } \\
\text { the } \\
\text { Onset }\end{array}$ & $\begin{array}{c}\text { Acute } \\
\text { Attack } \\
\text { Therapy } \\
\end{array}$ & $\begin{array}{l}\text { Clinical } \\
\text { Course }\end{array}$ & $\begin{array}{l}\text { Long-Term } \\
\text { Therapy }\end{array}$ & $\begin{array}{c}\text { EDSS at } \\
\text { Last } \\
\text { Follow-Up } \\
\end{array}$ \\
\hline 1 & $\mathrm{~F}, 16$ & $\begin{array}{l}\text { Headache, } \\
\text { vomiting, } \\
\text { vertigo }\end{array}$ & $\begin{array}{l}\text { Right centrum } \\
\text { semiovale, near the } \\
\text { trigone and the } \\
\text { temporal horn of the } \\
\text { right ventricle, pons, } \\
\text { medulla oblongata, } \\
\text { dorsal spine }\end{array}$ & Yes & $\begin{array}{l}\text { AQP4-IgG } \\
\text { positive }\end{array}$ & No & $\begin{array}{l}\text { Normal } \\
\text { VEPs, SEPs } \\
\text { and MEPs }\end{array}$ & 3 & IVMP & Monophasic & $\begin{array}{l}\text { Oral CS, } \\
\text { HCQ, MMF }\end{array}$ & 0 \\
\hline 2 & $\mathrm{~F}, 9$ & $\begin{array}{c}\text { Bilateral ON, } \\
\text { progressive } \\
\text { bilateral lower } \\
\text { limb } \\
\text { hyposthenia }\end{array}$ & $\begin{array}{l}\text { Right frontal } \\
\text { subcortical region, } \\
\text { corpus callosum, } \\
\text { cervical and dorsal } \\
\text { spine }\end{array}$ & Yes & $\begin{array}{l}\text { AQP4-IgG } \\
\text { positive }\end{array}$ & Yes & $\begin{array}{l}\text { Abnormal } \\
\text { VEPs and } \\
\text { SEPs (MEPs } \\
\text { not } \\
\text { available) }\end{array}$ & 4 & IVMP & Relapsing & $\begin{array}{l}\text { Oral CS, } \\
\text { RTX, AZA }\end{array}$ & 5 \\
\hline 3 & $\mathrm{~F}, 8$ & Bilateral ON & Bilateral optic nerves & $\begin{array}{c}\text { Yes, left } \\
\text { optic nerve }\end{array}$ & $\begin{array}{l}\text { AQP4-IgG } \\
\text { positive }\end{array}$ & No & $\begin{array}{l}\text { Abnormal } \\
\text { VEPs, } \\
\text { normal SEPs } \\
\text { and MEPs }\end{array}$ & 4 & IVMP, PE & Monophasic & RTX & 0 \\
\hline 4 & $\mathrm{M}, 15$ & $\begin{array}{l}\text { Progressive } \\
\text { right lower } \\
\text { limb paresis }\end{array}$ & $\begin{array}{l}\text { From cervical spine to } \\
\text { the conus medullaris }\end{array}$ & No & $\begin{array}{l}\text { AQP4-IgG } \\
\text { and } \\
\text { MOG-IgG } \\
\text { negative }\end{array}$ & No & $\begin{array}{l}\text { Abnormal } \\
\text { VEPs, SEPs } \\
\text { and MEPs }\end{array}$ & 8.5 & IVMP & Relapsing & $\mathrm{RTX}, \mathrm{MMF}$ & 0 \\
\hline 5 & $\mathrm{M}, 10$ & $\begin{array}{l}\text { Right lower } \\
\text { limb paresis, } \\
\text { sensory level at } \\
\text { T10-T11 }\end{array}$ & $\begin{array}{l}\text { Diffuse bilateral } \\
\text { cerebral involvement, } \\
\text { cervical spine }\end{array}$ & Yes & $\begin{array}{l}\text { AQP4-IgG } \\
\text { and } \\
\text { MOG-IgG } \\
\text { negative }\end{array}$ & No & $\begin{array}{l}\text { Abnormal } \\
\text { VEPs, SEPs } \\
\text { and MEPs }\end{array}$ & 9 & IVMP, PE & Relapsing & RTX & 7.5 \\
\hline 6 & $\mathrm{M}, 13$ & $\begin{array}{c}\text { Gait ataxia, } \\
\text { bilateral lower } \\
\text { limb } \\
\text { paresthesia, } \\
\text { pyramidal } \\
\text { signs, sensory } \\
\text { level at T10 }\end{array}$ & Dorsal spine & Yes & $\begin{array}{l}\text { AQP4-IgG } \\
\text { and } \\
\text { MOG-IgG } \\
\text { negative }\end{array}$ & No & $\begin{array}{c}\text { Abnormal } \\
\text { VEPs (SEPs } \\
\text { and MEPs } \\
\text { not } \\
\text { available) }\end{array}$ & 6 & IVMP & Monophasic & Oral CS & 0 \\
\hline 7 & $\mathrm{M}, 11$ & Unilateral ON & $\begin{array}{l}\text { Left optic nerve and } \\
\text { cervical spine }\end{array}$ & Yes & $\begin{array}{l}\text { AQP4-IgG } \\
\text { and } \\
\text { MOG-IgG } \\
\text { negative }\end{array}$ & No & $\begin{array}{l}\text { Abnormal } \\
\text { VEPs, } \\
\text { normal SEPs } \\
\text { and MEPs }\end{array}$ & 2 & IVMP & Relapsing & IVIG & 1 \\
\hline
\end{tabular}

Pt: patient; y: years; Gen: gender; CSF: cerebrospinal fluid; OCBs: oligoclonal bands; Gd: gadolinium; EDSS: Expanded Disability Status Scale; ON: optic neuritis; PE: plasma exchange; IVMP: intravenous methylprednisolone; CS: corticosteroids; MMF: mycophenolate mofetil; RTX: rituximab; HCQ: hydroxychloroquine; AZA: azathioprine; IVIG: intravenous immunoglobulin.

\subsection{NMOSDs with AQP4-IgG}

\subsubsection{Case 1}

Patient 1 is a 16-year-old female who reported headache, vomiting and vertigo. Magnetic resonance imaging (MRI) showed multiple T2-hyperintense lesions with gadolinium enhancement in the brain, brainstem, and dorsal spine (Figure 1A). Cerebrospinal fluid (CSF) studies showed an increased number of lymphocytes, in absence of oligoclonal 
bands (OCBs). Expanded Disability Status Score (EDSS) was 3 at this time. Diagnosis of NMOSD was confirmed by the positivity of AQP4-IgG [15]. Given the positivity of anti-Sjögren's-syndrome-related antigen A autoantibodies (anti-SSA/Ro), the presence of eye dryness, and the evidence of lung interstitiopathy at chest computed tomography (CT) and spirometry, a further diagnosis of Sjögren's syndrome was made. She has been treated with high-dose steroids, hydroxychloroquine, mycophenolate mofetil (MMF), and rituximab (RTX). One year after diagnosis, AQP4-IgG became negative. At the time of last follow-up, 41 months after diagnosis, MRI showed stability of preexisting lesions, with no gadolinium enhancement, and in absence of new lesions. EDSS was 0. She was daily taking prednisone, hydroxychloroquine, and MMF. AQP4-IgG remained negative.

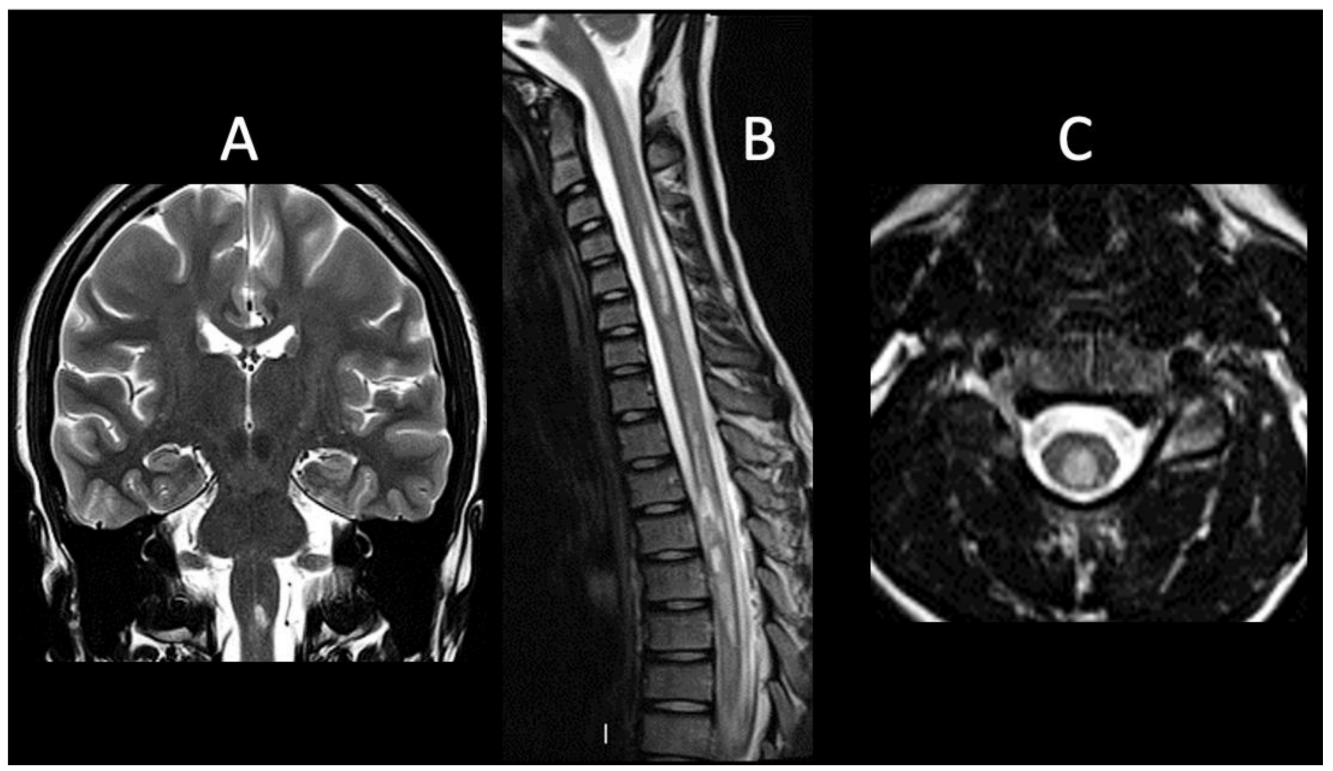

Figure 1. NMOSDs with AQP4-IgG. (A). Coronal T2-weighted image (WI) shows hyperintense lesion in brainstem (case 1), (B). sagittal T2-WI shows multiple and confluent hyperintense lesions in cervical and dorsal spine consistent with LETM (case 2), (C). axial T2-WI shows hyperintense lesion in cervical spine (case 2).

\subsubsection{Case 2}

Patient 2 is a female who presented at the age of 9 years with bilateral optic neuritis $(\mathrm{ON})$ and progressive hyposthenia of the lower limbs. MRI investigation revealed multiple T2-hyperintense lesions in the right frontal subcortical region, corpus callosum, and in the cervical and dorsal spine (Figure 1B,C). Diagnosis of NMOSD was then confirmed by the positivity of AQP4-IgG [15]. CSF OCBs were present. EDSS was four at the time of the first attack. Hematopoietic stem cell transplantation (HSCT) from the HLA-haploidentical father was performed, after several relapses due to an ineffective disease control (EDSS was 6.5) despite treatment with high-dose steroids, azathioprine (AZA), cyclosporine, and RTX. At the time of last follow-up, 42 months after HSCT, MRI showed a significant reduction of preexisting lesions with no gadolinium enhancement in absence of new lesions. The patient had no re-exacerbation, showing improvement in EDSS which became 5. AQP4-IgG remained positive.

\subsubsection{Case 3}

Patient 3 is an 8-year-old female who presented with bilateral ON (EDSS was 4). MRI showed T2-hyperintensity of both optic nerves, with gadolinium enhancement on the left side. Visual evoked potentials (VEPs) showed a bilateral increase in latency. CSF OCBs were negative. Diagnosis of NMOSD was then confirmed by the positivity of AQP4-IgG [15]. After initial steroid treatment, she underwent plasmapheresis, followed by RTX. At the last 
follow-up, 19 months after diagnosis, MRI showed a significant reduction of preexisting lesions, with the patient undergoing the third cycle of RTX in absence of re-exacerbations. EDSS was 0.

\subsection{NMOSDs without AQP4-IgG}

\subsubsection{Case 4}

Patient 4 is a male affected by ulcerative colitis since he was 4 . He underwent a subtotal colectomy at the age of 12, because of a poor disease control. At the age of 15, he presented with a progressive right lower limb paresis; MRI detected multiple T2-hyperintense lesions extending from the cervical spine to the conus medullaris, with no gadolinium enhancement (Figure 2A). Serum AQP4-IgG, MOG-IgG and CSF OCBs were negative. VEPs showed a bilateral increase in latency, in the absence of visual symptoms. Motor evoked potentials (MEPs) and somatosensory evoked potentials (SEPs) were abnormal. EDSS at the time of first attack was 8.5. He presented acute myelitis with longitudinally extensive transverse myelitis (LETM) and increased latency to VEPs, so a suspected NMOSD diagnosis was made [15]. After an initial response to steroids, six months later, he had a clinical and radiological relapse with a new cerebral T2-hyperintense lesion in the cerebral deep white matter and gadolinium-enhancement of previous spinal lesions. In particular, the inflammatory brain lesion was not typical for NMOSD [15]. Therefore, RTX was administered. Maintenance therapy with MMF was started and then discontinued at age 19 due to clinical stability. At the time of last follow-up, 60 months after diagnosis, MRI and electrophysiologic studies were normal, with an EDSS of 0.

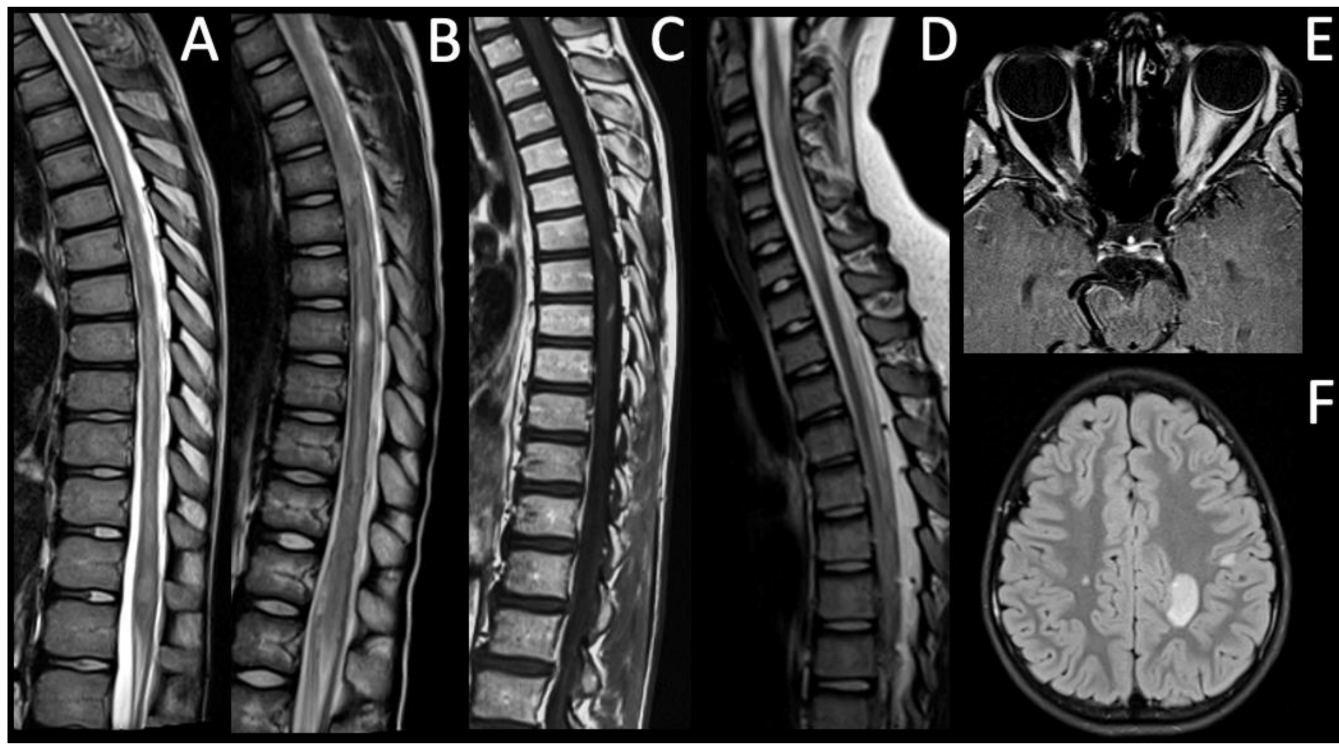

Figure 2. NMOSDs without AQP4-IgG. (A). Sagittal T2-WI shows multiple and confluent hyperintense lesions in the cervical and dorsal spine consistent with LETM (case 4), (B). sagittal T2-WI shows multiple and confluent hyperintense lesions in the dorsal spine extending from level T5 to the conus medullaris (case 5), (C). sagittal T1-WI acquired after the intravenous (iv) administration of gadolinium (gd) shows intramedullary enhancement between T6 and T8 levels (case 6), (D). sagittal T2-WI shows hyperintense lesions in the cervical spine (C2-C7) consistent with LTM (case 7), (E). axial T1-WI acquired after iv administration of gd shows left optic nerve enhancement consistent with left ON (case 7), (F) axial T2-FLAIR shows multiple brain hyperintense lesions (case 5).

\subsubsection{Case 5}

Patient 5 is a 10-year-old male who presented with a right lower limb paresis; sensory level was T10-T11. MRI showed multiple T2-hyperintense lesions with gadolinium enhancement in both cerebral hemispheres and in the cervical spine (Figure 2B,F). EDSS was 
9 at diagnosis. VEPs showed a bilateral increase in latency, especially on the left side. MEPs and SEPs indicated an impairment of motor and somatosensory pathways of lower limbs. Serum AQP4-IgG, MOG-IgG and CSF OCBs were negative. Serum and CSF serology for neurotropic pathogens was negative. After the initial treatment with high-dose steroids, he developed paraplegia and left upper limb paresis, urinary and fecal incontinence, with a sensory level at T5-T6. T2-hyperintense lesions had extended towards conus medullaris. Suspected seronegative NMOSD diagnosis was made [15].

Therefore, he underwent PLEX followed by RTX, with a partial clinical improvement. At the time of last follow-up, 63 months after diagnosis, he presented with paraplegia, scoliosis, neurogenic bladder, and bowel dysfunction. EDSS was 7.5.

\subsubsection{Case 6}

Patient 6 is a 13-year-old male who presented with gait ataxia, bilateral lower limb paresthesia, and pyramidal signs. Sensory level was T10. MRI showed hyperintense intramedullary signal at T2-weighted imaging between T5 and T9 (Figure 2C), with gadolinium enhancement. The patient did not report visual disturbances but had a concomitant bilateral increase in latency of VEPs that led to a suspected NMOSD diagnosis [15]; AQP4IgG and MOG-IgG have always been negative. EDSS was 6 at onset. CSF OCBs were negative. He has shown a good response to steroids, initially administered intra-venously and subsequently orally as chronic therapy. At the last follow-up, 24 months after diagnosis, MRI showed stability of preexisting spinal lesions. EDSS was 0 . Left eye function was recovered, while an increased latency of right VEPs persisted.

\subsubsection{Case 7}

Patient 7 is a 11-year-old male who presented with left ON. MRI showed T2-hyperintense lesions with gadolinium enhancement in left optic nerve (Figure 2E) and T2-hyperintense medullary lesions with gadolinium enhancement between $\mathrm{C} 2$ and $\mathrm{C} 7$ levels consistent with LETM (Figure 2D). The patient had no signs or symptoms related to spinal cord lesion. Serum AQP4-IgG, MOG-IgG and CSF OCBs were negative, but an increased number of lymphocytes were found at CSF analysis.

MEPs and SEPs were normal and VEPs showed an increased latency only on the left side. EDSS was 2 at onset. Seronegative NMOSD diagnosis was made [15]. He has been treated with high-dose steroids intravenously, and then with oral steroids but, approximately five months later, the patient presented with right ON. Maintenance therapy was started with 7 cycles of intravenous immunoglobulin (IVIG). At the last follow-up, 24 months after diagnosis, he presented clinical and radiological stability, EDSS was 0.

\section{Literature Review}

\subsection{Methods}

This is a narrative review in which we have identified studies describing cases of NMOSD in pediatric age.

The research was performed in the PUBMED database (last search was performed on 22th December 2021) with no limited period range. The following search terms were used to track articles: neuromyelitis optica and children.

Our review included original studies and case reports exclusively on pediatric patients with NMOSD.

We excluded all reviews and studies in which the sample included adult patients or pediatric and adult patients with NMOSD or those with other ADS of the CNS, such as ADEM, MS and MOG associated diseases.

\subsection{Results}

After the initial identification of 757 papers, we selected for the final analysis 41 manuscripts fulfilling our inclusion criteria. Table 2 shows the main characteristics of all studies selected for this review. 
Table 2. Main characteristics of all studies selected for this review.

\begin{tabular}{|c|c|c|c|c|c|c|c|c|c|c|c|}
\hline Reference & Study Type & $\mathbf{N}$ & $\begin{array}{l}\text { Age Range } \\
\text { (years) }\end{array}$ & Diagnosis & $\begin{array}{l}\mathrm{AQP4} \\
+\end{array}$ & $\begin{array}{l}\text { MOG } \\
+\end{array}$ & $\begin{array}{l}\text { Seronegative } \\
\text { NMOSD }\end{array}$ & $\begin{array}{c}\text { Diagnostic } \\
\text { Criteria }\end{array}$ & Symptoms at Onset & Treatment & Outcome \\
\hline $\begin{array}{c}\text { Fragoso YD } \\
\text { et al., 2014 } \\
{[16]}\end{array}$ & Case series & $\mathrm{n}=29$ & $\begin{array}{c}13 \pm 3.4 \\
(5-17)\end{array}$ & NMO & $\mathrm{n}=22$ & NA & $\begin{array}{l}\text { Negative }(\mathrm{n}=5) \\
\text { not performed } \\
(\mathrm{n}=2)\end{array}$ & $\begin{array}{l}\text { Wingerchuk } \\
\text { et al., } 2006\end{array}$ & $\begin{array}{l}\text { Myelitis }(n=12), \\
\text { ON }(n=8), \text { myelitis } \\
\text { and } \mathrm{ON}(\mathrm{n}=9)\end{array}$ & $\begin{array}{c}\text { AZA }(\mathrm{n}=29), \\
\text { AZA + PDN } \\
(\mathrm{n}=4), \text { AZA + } \\
\text { GA }(\mathrm{n}=3), \text { IVIG } \\
(\mathrm{n}=6), \text { PLEX } \\
(\mathrm{n}=2), \text { MTX }+ \\
\text { PDN }(\mathrm{n}=1)\end{array}$ & $\mathrm{NA}$ \\
\hline $\begin{array}{l}\text { Paolilo RB } \\
\text { et al., 2020 } \\
\text { [17] }\end{array}$ & $\begin{array}{l}\text { Retrospective } \\
\text { study }\end{array}$ & $\mathrm{n}=67$ & $10.2 \pm 3.6$ & NMOSD & $\mathrm{n}=67$ & NA & NA & $\begin{array}{l}\text { Wingerchuk } \\
\text { et al., } 2015\end{array}$ & $\begin{array}{c}\mathrm{ON}(\mathrm{n}=20), \mathrm{TM} \\
(\mathrm{n}=15) \text {, area } \\
\text { postrema syndrome } \\
(\mathrm{n}=11) \\
\text { simultaneous ON } \\
\text { and TM }(\mathrm{n}=6), \\
\text { ADEM }(\mathrm{n}=6), \\
\text { isolated } \\
\text { diencephalic } \\
\text { syndrome }(\mathrm{n}=1), \\
\text { isolated brainstem } \\
\text { syndrome }(\mathrm{n}=3), \\
\text { multifocal } \\
\text { syndromes }(\mathrm{n}=5)\end{array}$ & $\begin{array}{l}1 \text { DMT }(\mathrm{n}=41), 2 \\
\text { DMTs }(\mathrm{n}=12), 3 \\
\text { DMTs }(\mathrm{n}=7), 4 \\
\text { DMTs }(\mathrm{n}=2), 5 \\
\text { DMTs }(\mathrm{n}=1), \\
\text { untreated }(\mathrm{n}=4)\end{array}$ & $\begin{array}{c}\text { EDSS } \geq 3 \\
(\mathrm{n}=29) \text {, visual } \\
\text { impairment } \\
(\mathrm{n}=32), \text { motor } \\
\text { deficits }(\mathrm{n}=14), \\
\text { cognitive } \\
\text { impairment } \\
(\mathrm{n}=17)\end{array}$ \\
\hline $\begin{array}{l}\text { Absoud M } \\
\text { et al., } 2015 \text { [8] }\end{array}$ & $\begin{array}{l}\text { Retrospective } \\
\text { study }\end{array}$ & $\mathrm{n}=20$ & $10.5(2.9-16.8)$ & NMO & $\mathrm{n}=8$ & NA & $\mathrm{n}=12$ & $\begin{array}{l}\text { Wingerchuk } \\
\text { et al., } 2006\end{array}$ & $\begin{array}{c}\mathrm{ON}(\mathrm{n}=12), \mathrm{TM} \\
(\mathrm{n}=3), \mathrm{ON}+\mathrm{TM} \\
(\mathrm{n}=3), \mathrm{ADEM} \\
(\mathrm{n}=2)\end{array}$ & $\begin{array}{l}\text { Oral PDN, AZA, } \\
\text { RTX, IVIG, MMF, } \\
\text { mitoxantrone, } \\
\text { ofatumumab }\end{array}$ & $\begin{array}{c}\text { Visual } \\
\text { impairment } \\
(\mathrm{n}=10), \\
\text { wheelchair- } \\
\text { dependent } \\
(\mathrm{n}=3)\end{array}$ \\
\hline $\begin{array}{l}\text { Chitnis T } \\
\text { et al., 2016 } \\
\text { [18] }\end{array}$ & $\begin{array}{c}\text { Prospective } \\
\text { multicenter } \\
\text { database }\end{array}$ & $\mathrm{n}=38$ & $10.2 \pm 4.7$ & $\begin{array}{l}\text { NMO } \\
\text { NMOSD }\end{array}$ & $\mathrm{n}=24$ & NA & $\mathrm{n}=14$ & $\begin{array}{l}\text { Wingerchuk } \\
\text { et al., } 2006 \\
\text { Wingerchuk } \\
\text { et al., } 2015\end{array}$ & $\begin{array}{c}\text { Visual }(\mathrm{n}=21) \\
\text { motor }(\mathrm{n}=17) \\
\text { constitutional } \\
(\mathrm{n}=23) \text { symptoms }\end{array}$ & $\begin{array}{c}\text { RTX }(47 \%), \text { MMF } \\
(39 \%), \text { AZA } \\
(24 \%), \text { PLEX } \\
(39 \%)\end{array}$ & $\begin{array}{l}\text { Number of } \\
\text { attacks in first } \\
\text { two years } \\
(1.84 \pm 1.44)\end{array}$ \\
\hline $\begin{array}{l}\text { Khan TR et al., } \\
2020[19]\end{array}$ & Case report & $\mathrm{n}=1$ & 2 & NMOSD & $\mathrm{n}=1$ & NA & $\mathrm{n}=0$ & $\begin{array}{l}\text { Wingerchuk } \\
\text { et al., } 2015\end{array}$ & $\begin{array}{l}\text { TM (progressive } \\
\text { left-hand weakness, } \\
\text { gait instability) }\end{array}$ & $\begin{array}{l}\text { IVMP, PLEX, } \\
\text { RTX, MMF }\end{array}$ & $\begin{array}{c}\text { Several } \\
\text { exacerbations }\end{array}$ \\
\hline $\begin{array}{c}\text { Gmuca S } \\
\text { et al., 2017 } \\
{[20]}\end{array}$ & Case series & $\mathrm{n}=4$ & $11-15$ & NMOSD + SS & $\mathrm{n}=4$ & NA & $\mathrm{n}=0$ & $\begin{array}{l}\text { Wingerchuk } \\
\text { et al., } 2015\end{array}$ & $\begin{array}{c}\mathrm{TM}+\mathrm{ON}(\mathrm{n}=1) \\
\mathrm{ON}(\mathrm{n}=2) \\
\text { intractable emesis } \\
\text { and hiccups }(\mathrm{n}=1)\end{array}$ & $\begin{array}{l}\text { IVMP, RTX, CP, } \\
\text { PLEX, HCQ, } \\
\text { MMF }\end{array}$ & $\mathrm{NA}$ \\
\hline $\begin{array}{l}\text { Marass H et al., } \\
2013 \text { [21] }\end{array}$ & Case report & $\mathrm{n}=1$ & 5.67 & NMO & $\mathrm{n}=1$ & NA & $\mathrm{n}=0$ & $\begin{array}{l}\text { Krupp et al., } \\
2007\end{array}$ & ON & $\begin{array}{c}\text { IVMP, oral CS, } \\
\text { AZA }\end{array}$ & $\begin{array}{l}\text { Several relapses } \\
\text { with vision loss }\end{array}$ \\
\hline $\begin{array}{l}\text { Mahmood } \\
\text { NA et al., } \\
2011 \text { [22] }\end{array}$ & Case series & $\mathrm{n}=2$ & $10-15$ & NMO & $\mathrm{n}=2$ & NA & $\mathrm{n}=0$ & $\begin{array}{l}\text { Wingerchuk } \\
\text { et al., } 2006\end{array}$ & $\mathrm{TM}(\mathrm{n}=2)$ & $\begin{array}{l}\text { IVMP, oral CS, } \\
\text { IVIG, AZA, RTX }\end{array}$ & $\begin{array}{c}\text { Relapses (ON, } \\
\text { bowel and } \\
\text { bladder retention, } \\
\text { leg weakness) }\end{array}$ \\
\hline $\begin{array}{l}\text { Ikeda } \mathrm{A} \text { et al., } \\
2019 \text { [23] }\end{array}$ & $\begin{array}{l}\text { Retrospective } \\
\text { study }\end{array}$ & $\mathrm{n}=4$ & $8(3-12)$ & NMOSD & $\mathrm{n}=0$ & $\mathrm{n}=4$ & $\mathrm{n}=0$ & $\begin{array}{l}\text { Wingerchuk } \\
\text { et al., } 2015\end{array}$ & $\begin{array}{c}\mathrm{ON}(\mathrm{n}=2) \\
\text { extremity weak- } \\
\text { ness/paresthesia } \\
(\mathrm{n}=2)\end{array}$ & $\begin{array}{l}\text { IVMP, oral CS, } \\
\text { PLEX, AZA, } \\
\text { tacrolimus }\end{array}$ & $\begin{array}{l}\text { Several relapses, } \\
\text { but no sequelae }\end{array}$ \\
\hline $\begin{array}{l}\text { Chuquilin M } \\
\text { et al., 2016 } \\
\text { [24] }\end{array}$ & Case report & $\mathrm{n}=2$ & $3-3.5$ & NMO & $\mathrm{n}=1$ & NA & NA & $\begin{array}{l}\text { Wingerchuk } \\
\text { et al., 2015 }\end{array}$ & Blindness $(n=2)$ & $\begin{array}{l}\text { GA, AZA, CS, } \\
\text { IFN-beta1a, RTX, } \\
\text { PLEX }\end{array}$ & Several relapses \\
\hline $\begin{array}{l}\text { Lotze TE et al., } \\
2018 \text { [25] }\end{array}$ & $\begin{array}{l}\text { Retrospective } \\
\text { study }\end{array}$ & $\mathrm{n}=9$ & $14(1.9-16)$ & NMOSD & $\mathrm{n}=7$ & $\mathrm{NA}$ & NA & $\begin{array}{l}\text { Wingerchuk } \\
\text { et al., 2006 }\end{array}$ & $\begin{array}{l}\text { Visual, motor, and } \\
\text { mixed symptoms }\end{array}$ & $\begin{array}{l}\text { IVMP, IVIG, } \\
\text { PLEX, CP, MMF, } \\
\text { RTX, AZA, GA }\end{array}$ & $\begin{array}{c}\text { Relapsing- } \\
\text { remitting course } \\
\text { in all patients }\end{array}$ \\
\hline $\begin{array}{c}\text { Rostásy K } \\
\text { et al., 2013 } \\
\text { [26] }\end{array}$ & $\begin{array}{l}\text { Retrospective } \\
\text { study }\end{array}$ & $\mathrm{n}=8$ & $10.5(3-15)$ & NMO & $\mathrm{n}=2$ & $\mathrm{n}=3$ & $\mathrm{n}=3$ & $\begin{array}{l}\text { Wingerchuk } \\
\text { et al., 2006 }\end{array}$ & Various & $\begin{array}{l}\text { RTX, AZA, oral } \\
\text { CS, IVIG }\end{array}$ & $\begin{array}{c}\text { Monophasic } \\
(n=1) \text {, relapses } \\
(n=7)\end{array}$ \\
\hline $\begin{array}{l}\text { Dimitrijevic N } \\
\text { et al., 2012 } \\
{[27]}\end{array}$ & Case report & $\mathrm{n}=1$ & 3 & NMO & $\mathrm{n}=1$ & NA & NA & $\begin{array}{l}\text { Wingerchuk } \\
\text { et al., 2006 }\end{array}$ & $\begin{array}{c}\text { Isolated ON } \\
\text { (sudden visual loss) }\end{array}$ & $\begin{array}{c}\text { IVMP, oral CS, } \\
\text { IVIG }\end{array}$ & $\begin{array}{l}\text { Several relapses } \\
\text { leading to } \\
\text { blindness, } \\
\text { paraplegia, } \\
\text { urinary and } \\
\text { bowel } \\
\text { incontinence, } \\
\text { short stature }\end{array}$ \\
\hline $\begin{array}{l}\text { Loma IP et al., } \\
2008 \text { [28] }\end{array}$ & Case report & $\mathrm{n}=1$ & 3.9 & NMO & $\mathrm{n}=1$ & NA & $\mathrm{n}=0$ & $\begin{array}{l}\text { Wingerchuk } \\
\text { et al., } 2006\end{array}$ & $\begin{array}{c}\text { Bilateral leg } \\
\text { weakness, urinary } \\
\text { and bowel } \\
\text { incontinence } \\
\text { (LETM) }\end{array}$ & $\begin{array}{l}\text { IVMP, oral CS, } \\
\text { IVIG, AZA }\end{array}$ & $\begin{array}{l}\text { Several relapses, } \\
\text { but no sequelae }\end{array}$ \\
\hline $\begin{array}{l}\text { Yavuz H et al., } \\
2013 \text { [29] }\end{array}$ & Case report & $\mathrm{n}=1$ & 13 & NMO & NA & NA & NA & $\begin{array}{l}\text { Sellner et al., } \\
2010\end{array}$ & $\begin{array}{c}\text { Visual loss, } \\
\text { behavioral changes }\end{array}$ & $\begin{array}{l}\text { CS, IVIG, PLEX, } \\
\text { AZA }\end{array}$ & $\begin{array}{l}\text { Several relapses } \\
\text { with vision } \\
\text { impairment }\end{array}$ \\
\hline $\begin{array}{c}\text { Marino A } \\
\text { et al., 2017 } \\
{[30]}\end{array}$ & Case report & $\mathrm{n}=1$ & 1.9 & $\mathrm{NMO}+\mathrm{SS}$ & $\mathrm{n}=1$ & NA & $\mathrm{n}=0$ & $\begin{array}{l}\text { Wingerchuk } \\
\text { et al., 2015 }\end{array}$ & $\begin{array}{c}\text { Central pontine } \\
\text { myelinolysis, } \\
\text { unilateral ON, ON + } \\
\text { TM }\end{array}$ & $\begin{array}{c}\text { IVIG, IVMP, } \\
\text { PLEX, HCQ, CP, } \\
\text { RTX, MMF, TCZ }\end{array}$ & $\begin{array}{l}\text { Several relapses } \\
\text { before TCZ } \\
\text { introduction }\end{array}$ \\
\hline $\begin{array}{l}\text { He Det al., } \\
2014 \text { [31] }\end{array}$ & Case report & $\mathrm{n}=1$ & 5 & NMO & $\mathrm{n}=1$ & NA & $\mathrm{n}=0$ & $\begin{array}{l}\text { Krupp et al., } \\
2007\end{array}$ & Bilateral ON & $\begin{array}{l}\text { IVMP, oral CS, } \\
\text { IVIG, RTX }\end{array}$ & $\begin{array}{c}\text { Several relapses } \\
\text { before RTX } \\
\text { introduction }\end{array}$ \\
\hline $\begin{array}{l}\text { Elpers C et al., } \\
2015[32]\end{array}$ & Case report & $\mathrm{n}=1$ & 12 & NMO & $\mathrm{n}=1$ & NA & $\mathrm{n}=0$ & $\begin{array}{l}\text { Wingerchuk } \\
\text { et al., 2006 }\end{array}$ & $\begin{array}{l}\text { Left arm paresis, left } \\
\text { leg progressive } \\
\text { paresis, dizziness, } \\
\text { neck pain (LETM) }\end{array}$ & $\begin{array}{l}\text { IVMP, oral CS, } \\
\text { PLEX, AZA, RTX }\end{array}$ & $\begin{array}{l}\text { Relapses before } \\
\text { RTX introduction }\end{array}$ \\
\hline $\begin{array}{c}\text { Maillart E } \\
\text { et al., 2020 } \\
{[33]}\end{array}$ & Case report & $\mathrm{n}=1$ & 8 & NMOSD & $\mathrm{n}=1$ & NA & $\mathrm{n}=0$ & $\begin{array}{l}\text { Wingerchuk } \\
\text { et al., } 2015\end{array}$ & $\begin{array}{c}\text { Bilateral ON + } \\
\text { LETM }\end{array}$ & $\begin{array}{c}\text { IVMP, oral CS, } \\
\text { PLEX, MMF, } \\
\text { RTX, } \\
\text { ofatumumab }\end{array}$ & $\begin{array}{l}\text { Several relapses } \\
\text { with permanent } \\
\text { visual disability }\end{array}$ \\
\hline $\begin{array}{c}\text { Kamawal A } \\
\text { et al., 2019 } \\
\text { [34] }\end{array}$ & Case report & $\mathrm{n}=1$ & 6 & NMOSD & $\mathrm{n}=0$ & $\mathrm{n}=1$ & $\mathrm{n}=0$ & $\begin{array}{l}\text { Wingerchuk } \\
\text { et al., } 2015\end{array}$ & $\begin{array}{l}\text { Progressive } \\
\text { headache, } \\
\text { meningism }\end{array}$ & $\begin{array}{l}\text { IVMP, PLEX, } \\
\text { MMF }\end{array}$ & $\begin{array}{l}\text { Neurological } \\
\text { restitutio ad } \\
\text { integrum within } \\
\text { two months }\end{array}$ \\
\hline
\end{tabular}


Table 2. Cont.

\begin{tabular}{|c|c|c|c|c|c|c|c|c|c|c|c|}
\hline Reference & Study Type & $\mathbf{N}$ & $\begin{array}{l}\text { Age Range } \\
\text { (years) }\end{array}$ & Diagnosis & $\begin{array}{l}\mathrm{AQP4} \\
+\end{array}$ & $\begin{array}{c}\text { MOG } \\
+\end{array}$ & $\begin{array}{l}\text { Seronegative } \\
\text { NMOSD }\end{array}$ & $\begin{array}{c}\text { Diagnostic } \\
\text { Criteria }\end{array}$ & Symptoms at Onset & Treatment & Outcome \\
\hline $\begin{array}{l}\text { Hudson LA } \\
\text { et al., 2006 } \\
\text { [35] }\end{array}$ & Case report & $\mathrm{n}=1$ & 8 & NMO & $\mathrm{n}=1$ & NA & $\mathrm{n}=0$ & $\begin{array}{l}\text { Wingerchuk } \\
\text { et al., } 2006\end{array}$ & $\begin{array}{l}\text { Bilateral upper } \\
\text { extremity } \\
\text { paresthesia }\end{array}$ & IVMP, oral CS & One relapse \\
\hline $\begin{array}{l}\text { Gokce G et al., } \\
2013 \text { [36] }\end{array}$ & Case report & $\mathrm{n}=1$ & 10 & NMO & $\mathrm{n}=1$ & NA & $\mathrm{n}=0$ & $\begin{array}{l}\text { Wingerchuk } \\
\text { et al., } 2006\end{array}$ & Sudden visual loss & $\begin{array}{c}\text { IVMP, oral CS, } \\
\text { AZA }\end{array}$ & $\begin{array}{c}\text { Significant } \\
\text { bilateral optic } \\
\text { atrophy }\end{array}$ \\
\hline $\begin{array}{l}\text { Khan TR et al., } \\
2021[37]\end{array}$ & Case report & $\mathrm{n}=1$ & 2 & NMOSD & $\mathrm{n}=1$ & NA & $\mathrm{n}=0$ & $\begin{array}{l}\text { Wingerchuk } \\
\text { et al., } 2015\end{array}$ & $\begin{array}{l}\text { Left-hand weakness } \\
\text { and abnormal gait } \\
\text { (LETM) }\end{array}$ & $\begin{array}{c}\text { PLEX, RTX, } \\
\text { MMF, TCZ, } \\
\text { autologous } \\
\text { HSCT }\end{array}$ & $\begin{array}{c}\text { Highly active } \\
\text { disease with } \\
\text { several } \\
\text { hospitalizations }\end{array}$ \\
\hline $\begin{array}{l}\text { Arnold TW } \\
\text { et al., 1987 } \\
\text { [38] }\end{array}$ & Case report & $\mathrm{n}=1$ & 12 & NMO & NA & NA & NA & NA & $\begin{array}{l}\text { Decreased vision in } \\
\text { the right eye, } \\
\text { paraparesis, bilateral } \\
\text { leg pain }\end{array}$ & PDN & $\begin{array}{c}\text { Normal } \\
\text { neurologic } \\
\text { examination } \\
\text { eight months } \\
\text { after discharge }\end{array}$ \\
\hline $\begin{array}{l}\text { Davis R et al., } \\
1996[39]\end{array}$ & Case report & $\mathrm{n}=1$ & 4 & NMO & NA & NA & NA & NA & $\mathrm{TM}$ & $\begin{array}{c}\text { IV and oral } \\
\text { dexamethasone }\end{array}$ & $\begin{array}{c}\text { One relapse } \\
(\mathrm{ON}) ; \\
\text { unremarkable } \\
\text { neurologic } \\
\text { examination after } \\
18 \text { months }\end{array}$ \\
\hline $\begin{array}{l}\text { Peña JA et al., } \\
2011 \text { [40] }\end{array}$ & Cohort study & $\mathrm{n}=6$ & $11(5-13)$ & NMO & $\mathrm{n}=4$ & NA & $\begin{array}{l}\text { Negative }(\mathrm{n}=1) \\
\text { not performed } \\
(\mathrm{n}=1)\end{array}$ & $\begin{array}{l}\text { Wingerchuk } \\
\text { et al., } 2006\end{array}$ & $\begin{array}{c}\mathrm{ON}(\mathrm{n}=3), \mathrm{TM} \\
(\mathrm{n}=1), \mathrm{ON}+\mathrm{TM} \\
(\mathrm{n}=2)\end{array}$ & $\begin{array}{l}\text { IVMP, IVIG, } \\
\text { PDN, AZA, } \\
\text { IFN-beta1a }\end{array}$ & $\begin{array}{l}\text { Relapsing- } \\
\text { remitting course } \\
\text { with bilateral } \\
\text { vision loss and } \\
\text { paraparesis in all } \\
\text { patients }\end{array}$ \\
\hline $\begin{array}{l}\text { Numata Y } \\
\text { et al., 2015 } \\
\text { [41] }\end{array}$ & Case report & $\mathrm{n}=1$ & 9 & NMO & $\mathrm{n}=1$ & NA & $\mathrm{n}=0$ & $\begin{array}{l}\text { Wingerchuk } \\
\text { et al., } 2006\end{array}$ & $\begin{array}{l}\text { Nausea, vomiting, } \\
\text { intractable hiccups }\end{array}$ & $\begin{array}{l}\text { IVMP, IVIG, oral } \\
\text { PDN }\end{array}$ & $\begin{array}{l}\text { Recurrence-free } \\
\text { for } 10 \text { months } \\
\text { after discharge }\end{array}$ \\
\hline $\begin{array}{l}\text { Milani N. } \\
\text { et al., 2004 } \\
\text { [42] }\end{array}$ & Case report & $\mathrm{n}=1$ & 7 & NMO & NA & NA & NA & $\begin{array}{l}\text { Wingerchuk } \\
\text { et al., } 1999\end{array}$ & $\begin{array}{l}\text { Fever and vomiting, } \\
\text { followed by } \\
\text { headache, neck } \\
\text { stiffness and visual } \\
\text { impairment }\end{array}$ & $\begin{array}{l}\text { IVMP, IVIG, } \\
\text { PDN, CP, AZA }\end{array}$ & $\begin{array}{l}\text { Relapsing- } \\
\text { remitting course: } \\
\text { paraparesis with } \\
\text { normal brain } \\
\text { MRI }\end{array}$ \\
\hline $\begin{array}{l}\text { Longoni G } \\
\text { et al., 2014 } \\
\text { [43] }\end{array}$ & Case report & $\mathrm{n}=1$ & 10 & NMOSD & $\mathrm{n}=1$ & NA & $\mathrm{n}=0$ & NA & $\begin{array}{l}\text { Subacute lower limb } \\
\text { weakness, gait } \\
\text { ataxia }\end{array}$ & $\begin{array}{c}\text { Targeted } \\
\text { immunotherapy }\end{array}$ & $\begin{array}{l}\text { Partial lesion } \\
\text { resolution }\end{array}$ \\
\hline $\begin{array}{c}\text { Bianchi A } \\
\text { et al., 2017 } \\
\text { [44] }\end{array}$ & Case report & $\mathrm{n}=1$ & 7 & NMOSD & $\mathrm{n}=1$ & NA & $\mathrm{n}=0$ & $\begin{array}{l}\text { Wingerchuk } \\
\text { et al., } 2015\end{array}$ & $\begin{array}{l}\text { Cervical back pain, } \\
\text { paraparesis }\end{array}$ & $\begin{array}{l}\text { IVMP, oral CS, } \\
\text { AZA, RTX }\end{array}$ & $\begin{array}{l}\text { Three episodes of } \\
\text { isolated recurrent } \\
\text { myelitis with no } \\
\text { further relapses } \\
\text { after RTX } \\
\text { introduction }\end{array}$ \\
\hline $\begin{array}{l}\text { Arabshahi B } \\
\text { et al., 2006 } \\
\text { [45] }\end{array}$ & Case report & $\mathrm{n}=1$ & 11 & $\mathrm{NMO}+\mathrm{SS}$ & NA & NA & NA & NA & Bilateral ON & CS, PLEX, CP & $\underset{\text { impairment }}{\text { Visual }}$ \\
\hline $\begin{array}{l}\text { Zhang Z et al., } \\
2021[46]\end{array}$ & Case series & $\mathrm{n}=11$ & $7(5-13)$ & NMOSD & $\begin{array}{l}\mathrm{n}=2 \\
(\mathrm{n}=1: \\
\text { both } \\
\text { AQP4 } \\
\text { and } \\
\text { MOG) }\end{array}$ & $\begin{array}{l}\mathrm{n}=2 \\
(\mathrm{n}=1: \\
\text { both } \\
\text { AQP4 } \\
\text { and } \\
\text { MOG) }\end{array}$ & $\begin{array}{l}\text { Negative }(\mathrm{n}=5) \\
\text { not performed } \\
(\mathrm{n}=3)\end{array}$ & $\begin{array}{l}\text { Wingerchuk } \\
\text { et al., } 2015\end{array}$ & $\begin{array}{l}\text { ON }(n=4), \mathrm{ON}+ \\
\text { encephalopathy } \\
(\mathrm{n}=5) \text {, myelitis }+ \\
\text { encephalopathy } \\
(\mathrm{n}=2)\end{array}$ & NA & NA \\
\hline $\begin{array}{c}\text { Baghbanian } \\
\text { SM et al., 2019 } \\
{[47]}\end{array}$ & Case series & $\mathrm{n}=10$ & $13(8-17)$ & NMOSD & $\mathrm{n}=7$ & NA & NA & $\begin{array}{l}\text { Wingerchuk } \\
\text { et al., } 2015\end{array}$ & $\begin{array}{l}\text { ON }(\mathrm{n}=4), \mathrm{ON}+ \\
\mathrm{TM}(\mathrm{n}=1), \mathrm{LETM} \\
(\mathrm{n}=3), \operatorname{APS}(\mathrm{n}=2)\end{array}$ & $\begin{array}{l}\text { IVMP, PLEX } \\
(\mathrm{n}=5), \text { AZA, } \\
\operatorname{RTX}(\mathrm{n}=5)\end{array}$ & $\begin{array}{l}\text { Favorable: EDSS } \\
\text { before preventive } \\
\text { therapy was } 3 \\
\text { (range } 0-5 \text { ) and } \\
\text { decreased to } 2.5 \\
\text { (range } 0-5 \text { ) after } \\
\text { preventive } \\
\text { therapy }\end{array}$ \\
\hline $\begin{array}{l}\text { Dembinski K } \\
\text { et al., 2013 } \\
\text { [48] }\end{array}$ & Case report & $\mathrm{n}=1$ & 13 & NMO & $\mathrm{n}=1$ & NA & $\mathrm{n}=0$ & $\begin{array}{l}\text { Wingerchuk } \\
\text { et al., } 2006\end{array}$ & $\begin{array}{l}\text { Encephalopathy } \\
\text { with waxing and } \\
\text { waning mental } \\
\text { status, flat affect, } \\
\text { ophthalmoparesis, } \\
\text { with decreased } \\
\text { visual acuity and } \\
\text { color agnosia }\end{array}$ & IVIG, IVMP, RTX & Favorable \\
\hline $\begin{array}{l}\text { Farhat L et al., } \\
2018 \text { [49] }\end{array}$ & Case report & $\mathrm{n}=1$ & 17 & NMO & NA & NA & NA & NA & $\begin{array}{l}\text { Acute vision loss in } \\
\text { the left eye }\end{array}$ & $\begin{array}{l}\text { AZA, CS, RTX, } \\
\text { IGRT }\end{array}$ & $\begin{array}{c}\text { Secondary } \\
\text { hypogammaglob- } \\
\text { ulinemia } \\
\text { following RTX } \\
\text { administration }\end{array}$ \\
\hline $\begin{array}{l}\text { Nosadini M } \\
\text { et al., 2016 } \\
\text { [50] }\end{array}$ & $\begin{array}{l}\text { Multicenter } \\
\text { retrospective } \\
\text { study }\end{array}$ & $n=16$ & $1.8-15.3$ & $\begin{array}{l}\text { NMO } \\
\text { NMOSD }\end{array}$ & NA & NA & NA & $\begin{array}{l}\text { Wingerchuk } \\
\text { et al., } 2015\end{array}$ & $\begin{array}{c}\mathrm{ON}(\mathrm{n}=4), \mathrm{TM} \\
(\mathrm{n}=4), \mathrm{ON}+\mathrm{TM} \\
(\mathrm{n}=2), \mathrm{BD}(\mathrm{n}=3) \\
\mathrm{TM}+\mathrm{BD}(\mathrm{n}=2), \mathrm{ON} \\
+\mathrm{BD}(\mathrm{n}=1)\end{array}$ & $\begin{array}{c}\text { Before RTX, } \\
62.5 \% \text { had } \\
\text { received AZT, } \\
\text { MMF, or CP. } \\
\text { Then all } 16 \\
\text { patients had } \geq 2 \\
\text { RTX courses }\end{array}$ & $\begin{array}{l}6 \text { patients were } \\
\text { relapse-free, } \\
\text { although } 21 \\
\text { relapses occurred } \\
\text { in } 10 \text { patients }\end{array}$ \\
\hline $\begin{array}{l}\text { Lechner C } \\
\text { et al., 2020 } \\
\text { [51] }\end{array}$ & $\begin{array}{l}\text { Multicenter pro- } \\
\text { and retrospective } \\
\text { study }\end{array}$ & $\mathrm{n}=24$ & $11(3-17)$ & NMOSD & $\mathrm{n}=16$ & $\mathrm{n}=4$ & $\mathrm{n}=3$ & $\begin{array}{l}\text { Wingerchuk } \\
\text { et al., } 2015\end{array}$ & $\begin{array}{c}\text { LETM }(\mathrm{n}=5), \text { BS } \\
(\mathrm{n}=3), \text { bilateral ON } \\
(\mathrm{n}=1), \text { APS }(\mathrm{n}=1), \\
\text { LETM + bilateral } \\
\text { ON }(\mathrm{n}=4), \mathrm{LETM}+ \\
\text { BS }(\mathrm{n}=2), \text { LETM }+ \\
\text { unilateral ON } \\
(\mathrm{n}=1), \mathrm{LETM}+\mathrm{BS}+ \\
\text { bilateral ON }(\mathrm{n}=1)\end{array}$ & $\begin{array}{c}\text { Acute: IVM, } \\
\text { IVIG }(\mathrm{n}=7), \\
\text { PLEX }(\mathrm{n}=7), \\
\text { RTX }(\mathrm{n}=2) . \\
\text { Long-term: RTX } \\
(\mathrm{n}=8), \mathrm{AZA} \\
(\mathrm{n}=4), \mathrm{TCZ} \\
(\mathrm{n}=2), \mathrm{MMF} \\
(\mathrm{n}=1), \mathrm{IVIG} \\
(\mathrm{n}=1), \mathrm{CP}(\mathrm{n}=1)\end{array}$ & NA \\
\hline
\end{tabular}


Table 2. Cont.

\begin{tabular}{|c|c|c|c|c|c|c|c|c|c|c|c|}
\hline Reference & Study Type & $\mathbf{N}$ & $\begin{array}{l}\text { Age Range } \\
\text { (years) }\end{array}$ & Diagnosis & $\begin{array}{l}\mathrm{AQP} 4 \\
+\end{array}$ & $\begin{array}{c}\text { MOG } \\
+\end{array}$ & $\begin{array}{l}\text { Seronegative } \\
\text { NMOSD }\end{array}$ & $\begin{array}{c}\text { Diagnostic } \\
\text { Criteria }\end{array}$ & Symptoms at Onset & Treatment & Outcome \\
\hline $\begin{array}{l}\text { Longoni G } \\
\text { et al., 2014 } \\
\text { [52] }\end{array}$ & $\begin{array}{l}\text { Retrospective } \\
\text { observational } \\
\text { cohort study }\end{array}$ & $\mathrm{n}=5$ & $10.9-17$ & $\begin{array}{l}\text { NMO } \\
\text { NMOSD }\end{array}$ & $\mathrm{n}=5$ & NA & $\mathrm{n}=0$ & NA & $\begin{array}{l}\text { ON }(\mathrm{n}=1) \text {, hiccups } \\
\text { with nausea and } \\
\text { vomiting }(\mathrm{n}=2) \text {, gait } \\
\text { disturbances }(\mathrm{n}=1) \text {, } \\
\text { hiccups with nausea } \\
\text { and vomiting and } \\
\text { progressive } \\
\text { encephalopathy } \\
(\mathrm{n}=1)\end{array}$ & $\begin{array}{l}\text { Acute: IVMP, } \\
\text { IVIG }(\mathrm{n}=3), \text { RTX } \\
\text { (at } 6 \text { months and } \\
12 \text { months) }\end{array}$ & $\begin{array}{l}\text { Favorable: EDSS } \\
\text { score in the } 5 \\
\text { patients } \\
\text { decreased from } \\
3.0 \text { at initiation of } \\
\text { RTX to } 2.0 \text { at } 6 \\
\text { months from } \\
\text { onset and } 0.8 \text { at } \\
12 \text { months from } \\
\text { onset }\end{array}$ \\
\hline $\begin{array}{c}\text { Camera V } \\
\text { et al., 2021 } \\
\text { [53] }\end{array}$ & $\begin{array}{l}\text { Retrospective } \\
\text { multicenter } \\
\text { cohort study }\end{array}$ & $\mathrm{n}=49$ & $12 \pm 4.1$ & NMOSD & $\mathrm{n}=49$ & NA & $\mathrm{n}=0$ & NA & $\begin{array}{c}\text { Multifocal onset } \\
\text { presentation }(26.5 \%), \\
\text { optic nerve }(47 \%), \\
\text { area } \\
\text { postrema/brainstem } \\
(48.9 \%) \text {, encephalon } \\
(28.6 \%)\end{array}$ & NA & NA \\
\hline $\begin{array}{c}\text { Bradshaw MJ } \\
\text { et al., 2017 } \\
\text { [54] }\end{array}$ & Case report & $\mathrm{n}=1$ & 3 & NMO & $\mathrm{n}=1$ & NA & $\mathrm{n}=0$ & $\begin{array}{l}\text { Wingerchuk } \\
\text { et al., } 2015\end{array}$ & $\begin{array}{l}\text { Progressive bilateral } \\
\text { vision loss, left } \\
\text { Babinski sign }\end{array}$ & $\begin{array}{l}5 \text { sessions of } \\
\text { PLEX/IVMP, } \\
\text { RTX }\end{array}$ & $\begin{array}{l}\text { Favorable: vision } \\
\text { improvement } \\
\text { after IVMP and } \\
\text { after first cycle of } \\
\text { RTX }\end{array}$ \\
\hline $\begin{array}{l}\text { Ceglie G et al., } \\
2019 \text { [55] }\end{array}$ & Case report & $\mathrm{n}=1$ & 9 & NMO & $\mathrm{n}=1$ & NA & $\mathrm{n}=0$ & NA & $\begin{array}{l}\text { Bilateral ON and } \\
\text { progressive } \\
\text { hyposthenia at the } \\
\text { lower limbs }\end{array}$ & $\begin{array}{l}\text { High-dose CS, } \\
\text { AZA, } \\
\text { cyclosporine, } \\
\text { RTX, allogeneic } \\
\text { HSCT }\end{array}$ & $\begin{array}{l}\text { Favorable: no } \\
\text { re-exacerbation, } \\
\text { with long-term } \\
\text { stabilization }\end{array}$ \\
\hline
\end{tabular}

NMO: neuromyelitis optica; NMOSD: neuromyelitis optica spectrum disorder; SS: Sjogren's syndrome; MOG: myelin oligodendrocyte glycoprotein; AQP4: aquaporin-4; +: positive; ON: optic neuritis; TM: transverse myelitis; ADEM: acute disseminated encephalomyelitis; LETM: longitudinally extensive transverse myelitis; APS: area postrema syndrome; BD: brainstem disease; BS: brainstem syndrome; IVMP: intravenous methylprednisolone; CS: corticosteroid; AZA: azathioprine; PDN: prednisone; GA: glatiramer acetate; IVIG: intravenous immunoglobulin; PLEX: plasma exchange; MTX: methotrexate; RTX: rituximab; DMT: disease modifying therapy; MMF: mycophenolate mofetil; CP: cyclophosphamide; HCQ: hydroxychloroquine; IFN-beta1a: interferon-beta1a; TCZ: tocilizumab; IGRT: immunoglobulin replacement therapy; HSCT: hematopoietic stem cell transplantation; EDSS Expanded Disability Status Scale; MRI: magnetic resonance imaging; NA: not available.

\section{Discussion}

\subsection{Epidemiology}

Pediatric patients with NMOSD are older than children with ADEM but about the same age as those with MS $[10,18]$. Moreover, they have a worse prognosis than those affected by MS, as reported by Tillema et al. [55]. Further, Yara Dadalti Fragoso et al. showed that morbidity in patients with early onset NMOSD was higher than in those with early onset MS, reporting that $40 \%$ of their patients had achieved an EDSS of 7 or more within 6 years [16]. According to most reports NMOSD is more common in females than men $[10,18]$, and the prevalence varies by ethnicity, being greater in non-Caucasian than in Caucasian populations [56].

In a large study conducted at the Mayo Clinic [10] in pediatric patients with NMOSD, median age at symptom onset was 12 years (range 4-18), there was a clear female preponderance ( $88 \%$ were girls) and mixed ethnic background.

In another US pediatric population study [18], mean patient age at symptom onset was $10.2 \pm 4.7$ years. In patients $<11$ years of age female/male ratio was $1.5 / 1$ while in those $>11$ years was 3.25/1. As for ethnicity, 37\% were African American, $11 \%$ were Asian and $13 \%$ of patients had Hispanic/Latino ethnicity.

In a Brazilian study of children with NMOSD the median age at onset was 13 years (range 5-17), female/male ratio was 2.6/1, and the preponderance of patients were of mixed ethnicity (Caucasian and African) [16].

In our series, the median age of presentation was 12 years (range 8-16), all AQP4-IgG positive patients were female and all seronegative NMOSD patients were male. Six patients were Caucasian, and one was non-Caucasian.

\subsection{Diagnostic Criteria}

For many years, Neuromyelitis Optica (NMO), previously called Devic's disease, has been identified as a subtype of MS. In 2004, with the discovery of a disease specific NMO-IgG antibody against the AQP4 water channel (AQP4-IgG), NMO was considered as a different autoimmune disease entity [1,57]. 
In 2006, Wingerchuk and colleagues [58] proposed the diagnostic criteria for NMO that require $\mathrm{ON}$, transverse myelitis (TM), and at least two of three supportive criteria: contiguous spinal cord MRI lesion extending over three or more segments, brain MRI nondiagnostic for MS, or AQP4-IgG seropositivity. In 2015, new diagnostic criteria were created using nomenclature that defines the term NMOSD and stratifies patients by serologic testing in NMOSD with and without AQP4-IgG [15].

According to these criteria, six core clinical characteristics for diagnosis of NMOSD were identified, including ON, TM, area postrema syndrome, acute brainstem syndrome, symptomatic narcolepsy or acute diencephalic clinical syndrome, and symptomatic cerebral syndrome. For diagnosis in AQP4-IgG positive NMOSD patients, only one core criterion is enough. For seronegative NMOSD patients, two core criteria are necessary for diagnosis occur as a consequence of one or more clinical events, and at least one of these two core criteria should be ON, TM with longitudinally extensive transverse myelitis (LETM), or area postrema syndrome. Moreover, in seronegative NMOSD patients are necessary, dissemination in space (two or more different core clinical characteristics) with additional MRI requirements specific for each clinical syndrome.

These diagnostic criteria have been validated in the pediatric group [18].

\subsection{Clinical Features}

The most frequent presenting features of NMOSD are visual, motor, sensory and constitutional symptoms (such as vomiting, fever, and seizures). In most pediatric reports of NMOSD, ON occurred as the first clinical event in $50-75 \%$ of patients and TM in $30-50 \%$, either alone or in combination [11]. Other symptoms are represented by ataxia, encephalopathy, and cranial nerve dysfunction, such as ophthalmoparesis or area postrema syndrome [59].

ON is defined as inflammation of the optic nerve and typically presents as loss of visual acuity accompanied by pain with eye movements and may be unilateral or bilateral. ON in NMOSD is commonly bilateral, longitudinally extensive, with a predilection for posterior optic nerve segments, particularly the optic chiasm $[54,60]$.

Another typical clinical feature is TM, defined as a spinal cord inflammation causing sensory, motor, and autonomic disorders. LETM consists of contiguous inflammatory lesions extending over three or more vertebral segments [61]. The clinical presentation of a patient with TM or LETM consists of para or tetraplegia, depending on the spinal cord level involved and with a sensory and sphincter dysfunction [62]. In pediatric patients, LETM is less specific for NMOSD and may be present in children with MS or ADEM [63].

Area postrema syndrome is one of the core clinical characteristics for NMOSD, due to the involvement of this circumventricular organ located in the caudal part of the fourth ventricle $[25,27,64]$. It presents with intractable vomiting, nausea, and hiccups secondary to inflammation in the emetic reflex center situated in this area [62].

Another core criterion is acute brainstem syndrome reported in $40 \%$ of pediatric NMOSD patients [62]. The involvement of this region causes a dysfunction of cranial nerves (facial palsy, trigeminal autonomic cephalalgia). In most cases, an involvement of the oculomotor nerves which presents with diplopia or nystagmus can be observed [63].

Other clinical events are dysarthria, vestibular ataxia, or the involvement of the respiratory center [65].

The involvement of thalamus/hypothalamus could be observed in these patients and could cause acute diencephalic clinical syndrome. Hypotension, hypersomnia, behavioral changes, amenorrhea galactorrhea syndrome and narcolepsy are manifestations that can occur with the diencephalic involvement [62]. Syndrome of inappropriate antidiuretic hormone secretion (SIADH) is a common clinical presentation, characterized by excessive release of antidiuretic hormone $(\mathrm{ADH})$ causing hyponatremia and is responsible for symptoms such as nausea, vomiting, irritability and in severe cases, seizures, and coma [66]. There are no data on the incidence of these symptoms in the pediatric population. 
Other rare neurological presentations in NMOSD are represented by cerebral syndromes that are reported in 16-32\% of AQP4-IgG positive children [8]. This ADEM-like phenotype has been reported in pediatric patients positive for MOG-IgG or AQP4-IgG [67]. A large cerebral hemispheric lesion could cause hemiparesis, visual field involvement, and signs of encephalopathy [8].

About $40 \%$ of pediatric patients with NMOSD have comorbid autoimmune diseases [54], including organ-specific disorders (e.g., myasthenia gravis, thyroid disease, ulcerative colitis, celiac disease, primary sclerosing cholangitis and idiopathic thrombocytopenic purpura) and non-organ-specific disorders (e.g., systemic lupus erythematosus, Sjögren syndrome and antiphospholipid syndrome) $[27,68]$.

One patient of ours with AQP4-IgG positive NMOSD (case 1) also had a diagnosis of Sjögren Syndrome. There are different case series that reported the association between pediatric NMOSD and Sjögren Syndrome [20,69].

According to recent studies, NMOSD can coexist with anti-N-methyl-D-aspartate receptor (NMDAR) encephalitis [70]. Anti-NMDAR encephalitis should be considered when NMOSD patients show atypical symptoms (abnormal behavior, psychiatric manifestations, autonomic dysfunction), or atypical brain lesions [71].

\subsection{Imaging Findings}

MRI is important in the diagnosis and follow-up of patients with NMOSD. ON is typically characterized as bilateral and longitudinally extensive over $1 / 2$ optic nerve length, frequently affecting posterior optic pathway, particularly the optic chiasm [72].

During the acute and subacute phases, optic nerve MRI shows hyperintensity in T2-weighted sequences and gadolinium enhancement in T1 weighted-images, while optic nerve atrophy and variable hyperintensity on T2-weighted images are observed in chronic stages [73]. In NMOSD pediatric patients with ON can be observed infraorbital fat gadolinium enhancement [11].

LETM is highly suggestive of NMOSD, usually characterized by extensive centrally located hyperintensity in T2 weighted images extending over three or more vertebral segments and involving both white and gray matter [74]. Cervical and thoracic spinal segments are typically compromised [74]. In the acute phase LETM is characterized by cord swelling and gadolinium enhancement in T1-weighted sequences, while in the chronic phase the spinal cord may appear atrophic [75]. In children, LETM is less specific for NMOSD than in adults and may be observed also in patients with MS, ADEM and monophasic TM [60,76,77].

Brain lesions are typically localized in areas of high AQP4 expression, such as circumventricular organs around the third and fourth ventricles (diencephalon and brainstem), including hypothalamic, thalamic and the periaqueductal area, supratentorial and infratentorial white matter, midbrain, ependymal surfaces of the cerebellum and corpus callosum [78]. Lesions in the corpus collosum are often large and follow the ependymal line with various shapes, different from those typical of MS, that are small and ovoid lesions [78]. The involvement of area postrema is one of the most typical brain features in patients with NMOSD [78].

During acute phases, brain lesions show hyperintensity on FLAIR/T2-weighted images and variable enhancement on T1-weighted images [79]. Gadolinium enhancement may be seen in approximately $30 \%$ of pediatric patients with brain lesions, usually with a cloud-like pattern of enhancement, as in adults $[8,10]$. Although the brain areas involved are the same in adult and pediatric patients, lesions are usually more common and larger $(>2 \mathrm{~cm})$ in children than in adults $[8,10]$.

\subsection{Laboratory Features}

The AQP4-IgG is a specific serum marker for NMOSD [42], described for the first time in 2004 by Lennon et al. [58]. The International Panel for NMO Diagnosis (IPND) recommended testing with a cell-based serum assay which has estimated sensitivity and 
specificity of 76.7\% and 99.8\%, respectively [15]. Testing for AQP4-IgG should be performed on serum, as it is more sensitive than CSF analysis. Data indicate significantly higher serum AQP4-IgG titer than CSF, as AQP4-IgG is produced in peripheral lymphoid tissues rather than intrathecally [80]. In a US study of pediatric NMOSD $65 \%$ of children were positive for AQP4-IgG and some patients became seropositive more than 3 years after onset [18]. Similar to adults, seropositivity for AQP4-IgG in children is usually associated with a relapsing course [76]. It is recommended to provide the dosage during the attack and before immunotherapy, because it may cause conversion to the negative state [80,81].

Children with NMOSD should also be tested for MOG-IgG. MOG-IgG has been found in a subgroup of adult and pediatric seronegative NMOSD patients [26]. Several studies have demonstrated that NMOSD patients with MOG-IgG usually have a younger age at presentation, fewer relapses, better outcomes, and are less frequently female than AQP4IgG positive patients $[7,82]$. MOG-IgG have also been associated with other ADS, including ADEM, monophasic or recurrent ON, ON following an ADEM onset (ADEM-ON) and TM [7]. The development of a sensitive and specific test for MOG-IgG has permitted the assessment of the clinical and radiological characteristics of patients $[83,84]$. Our case series did not include NMOSD patients with MOG-IgG.

CSF OCBs, a hallmark of MS, are absent in most NMOSD patients. According to some studies, OCBs in NMOSD patients are present in $15-30 \%$ of cases $[85,86]$, sometimes transiently detectable at the time of an attack [86]. During acute attacks of NMOSD pleocytosis is common, present in around 50\% of samples and is useful in distinguishing NMOSD from MS. Typically cell analysis demonstrates neutrophils, eosinophils and activated lymphocytes [61].

\subsection{Treatment}

\subsubsection{Acute Treatment}

NMOSD therapy includes the management of acute attacks and preventive treatment. All patients with suspected NMOSD should be treated in the acute phase because relapses can cause permanent disability [87]. Given the absence of controlled studies, in children treatment should performed based on the experiences in adults. A common approach to therapy begins with high dose intravenous methylprednisolone (IVMP). The recommended daily dose is $20 \mathrm{mg} / \mathrm{kg}$ / day to a maximum of $1000 \mathrm{mg}$ for 5 consecutive days, followed by a gradual decrease to oral glucocorticoid and long-term maintenance [88]. If the initial response to steroids is insufficient, then PLEX should be considered [89]. Treatment with PLEX consists of 5 exchanges over 5-10 days [90]. In their study, Abboud et al. found that PLEX + IVMP was associated with improved outcomes compared to IVMP alone, especially in patients taking preventive treatment [91]. If PLEX is not possible for contraindications, IVIG (a total dose of $2 \mathrm{~g} / \mathrm{kg}$ ) is recommended [56]. However, the efficacy of IVIG is uncertain; indeed, only a few studies have evaluated the effect of IVIG on acute exacerbation of NMOSD [92,93]. In the acute phase all patients in our cohort underwent treatment with IVMP, while only two also required therapy with PLEX.

There are no evidence-based guidelines for the acute treatment of children with MOGIgG. Usually, it consists of IVMP (20 mg/ kg/day, maximum $1 \mathrm{~g}$ for 3-5 days), IVIG (total of $2 \mathrm{~g} / \mathrm{kg}$ ) and PLEX administered individually or in combination, depending on age and clinical presentation [94].

\subsubsection{Preventive Treatment}

Preventive treatment is usually started after the first attack due to the risk of severe disability associated with each relapse. The rationale for the use of immunosuppressive therapy in pediatric patients is based on the experience in adults, in which preventive agents reduce the frequency and severity of relapses [18,95]. Practice generally recommends at least 5 years of maintenance therapy [96]. The current therapy options in pediatric patients include the off-label use of IVIG, AZA, MMF and RTX. 
Intravenous Immunoglobulin

IVIG has a potential role in treatment of NMOSD both for therapy of acute attacks and as preventive therapy [97]. It is an efficacious therapy in other antibody-mediated neurological diseases [57]. It is still unknown the exact anti-inflammatory mechanism of action of IVIG. Probably, IVIG acts on the immune system through various mechanisms, such as neutralization of autoantibodies, binding of the antibody target, inhibition of dendritic cell activation and leukocyte migration, complement inhibition, and blocking of Fc $\gamma$ receptors [98].

There are few studies in favor of the use of IVIG as preventive therapy in NMOSD. In a small study six NMO/NMOSD patients treated with 2 to 3 monthly IVIG infusions experienced a reduced number of relapses [98]. Another study reported successful reduction in relapse rates and good safety and tolerance in eight NMOSD patients treated with IVIG as preventive therapy [99]. In our series, one patient with seronegative NMOSD (case 7) is undergoing chronic therapy with cycles of IVIG, with good control of the disease.

\section{Azathioprine}

AZA is an immunosuppressive agent that acts through its effects as an antagonist of purine metabolism, resulting in the inhibition of DNA, RNA, and protein synthesis and consequently inhibition of cell proliferation, especially $\mathrm{T}$ and B cells [100]. A study of adult and pediatric AQP4-IgG NMOSD patients reported a modest efficacy of AZA [101]. Eighty-nine per cent of patients reported reduction in relapse rates and $61 \%$ remained relapse free at a median follow-up of 18 months with $2-3 \mathrm{mg} / \mathrm{kg} /$ day of AZA, but at last follow-up, treatment was discontinued in $46 \%$ due to adverse events. AZA treatment can have frequent side effects which may lead to intolerance. Common adverse events are nausea, vomiting, diarrhea, fever, thrombocytopenia, hepatotoxicity, leukopenia, and infections. One patient of ours with AQP4-IgG positive NMSOD (case 2) was treated with AZA with poor disease control.

\section{Mycophenolate Mofetil}

MMF is metabolized in the liver to the active mycophenolic acid. It is an inhibitor of inosine monophosphate dehydrogenase involved in guanosine nucleotide synthesis, used in the proliferation of B and T lymphocytes [102]. MMF is largely used in a variety of autoimmune diseases, included adult and pediatric NMOSD patients. In their observational study with NMOSD patients, Huh et al. reported that MMF therapy induced reduction of relapses, stabilized or improved disability, and was well tolerated [103]. In a retrospective study of 24 patients, $79 \%$ of NMOSD patients treated with MMF (median dose of $2000 \mathrm{mg}$ per day) reported reduction in relapse rates and, in $91 \%$ of patients, reduction or stabilization of disability [104]. In pediatric age the recommended dose is $600 \mathrm{mg} / \mathrm{m}^{2} / \mathrm{dose}$ twice a day [105].

\section{Rituximab}

RTX is an anti-CD20 monoclonal antibody that causes B cell depletion by binding to the CD20 antigen of B cell lymphocytes [106].

Several studies on children and adults with NMOSD have demonstrated that RTX induces reduction in the annualized relapse rate, it is well-tolerated and stabilizes or improves neurologic disability [50,52,106,107].

The recommended starting dose in pediatric patients is $375 \mathrm{mg} / \mathrm{m}^{2}$ weekly for 4 consecutive weeks or $500 \mathrm{mg} / \mathrm{m}^{2} /$ dose (max $1 \mathrm{~g}$ ), two infusions, 2 weeks apart [107], with monitoring of CD19 cell counts [50].

Common side effects include rash, a flu-like syndrome, headache, nausea, and fatigue, especially with the first infusion [50]. To reduce these reactions, pretreatment with acetaminophen, diphenhydramine and corticosteroids is recommended [108].

Patients should be screened also for hypogammaglobulinemia before and after administration of RTX, due to the risk of prolonged hypogammaglobulinemia and associated 
infections [106,109]. We had two patients, one with positive NMOSD (case 1) and the other seronegative (case 4) treated with MMF associated with RTX that showed a good control of disease. One patient with positive NMOSD (case 3) had a good response to RTX with an improvement in neurological disability, while another seronegative patient (case 5) had a partial response to RXT. Only one patient with AQP4-IgG (case 2) had no disease control after treatment with RTX.

Preventive Treatment in MOG-IgG Positive Patients

In MOG-IgG positive patients, chronic maintenance therapy is reserved for those with relapsing disease [94]. In a large retrospective multicenter study of adult and pediatric patients with MOG-IgG associated disorder, IVIG therapy was associated with the greatest reduction in relapse rate, as compared to other maintenance therapies (RTX, AZA, MMF) [99]. In a multinational European cohort study of 102 children, AZA, MMF, RTX, and particularly IVIG were associated with a reduction in relapse frequency [110]. In this study as well, maintenance with IVIG was found superior to other therapies.

\subsubsection{New Therapeutic Agents}

Several new therapeutic agents for NMOSD are being evaluated in trials in adult patients.

\section{Eculizumab}

Eculizumab is an anti-C5 monoclonal antibody. In a randomized double-blind placebocontrolled trial of 143 adult with AQP4-IgG-positive NMOSD, treatment with intravenous eculizumab reduced the risk of relapse [111]. Eculizumab is generally well tolerated. The most frequent side effects included upper respiratory tract infections, headache, diarrhea, and nausea [111]. The most severe adverse event is the increased risk of meningococcal and encapsulated bacterial infection [112]. For this reason, patients should be immunized with meningococcal vaccines. A trial that evaluates the safety and efficacy of eculizumab in pediatric patients with relapsing NMOSD is ongoing [113].

\section{Tocilizumab}

Tocilizumab is a monoclonal antibody against the IL-6 receptor. Based on different studies, it is a promising therapeutic option for NMOSD. Araki M. et al. reported that tocilizumab induced reduction of annualized relapse rate. Their study showed the efficacy and good safety of tocilizumab [114].

\section{Satralizumab}

As tocilizumab, satralizumab is a recombinant monoclonal antibody targeting interleukin-6 receptors [115]. In a phase three, randomized, double-blind, placebo-controlled trial with seronegative and seropositive NMOSD patients, satralizumab had a greater effect than placebo on reduction of the relapse rate, with a better response in AQP4-IgG seropositive patients [115]. There is an ongoing trial that evaluate the safety and efficacy of satralizumab in pediatric participants with NMOSD [116].

Inebilizumab

Inebilizumab is an anti-CD19 monoclonal antibody. Compared with anti-CD20 antibodies that deplete a small subset of B lymphocytes, anti-CD19 antibodies deplete a wider range of lymphocytes derived from the B-cell lineage [117]. In a trial with 230 seronegative and seropositive NMOSD patients, inebilizumab reduced the risk of relapse, disability, and MRI activity [118].

\section{Hematopoietic Stem Cell Transplantation}

The treatment of refractory NMOSD remains a considerable challenge for neurologists. In these NMOSD patients, Hematopoietic Stem Cell Transplantation (HSCT), both 
autologous (au HSCT) and allogeneic (al HSCT) HSCT should be considered as a possible therapeutic option in the most severe form of NMOSD [119]. Ceglie et al. reviewed the HSCT applications in NMOSD patients. Although the experience is limited, they observed that al HSCT is superior in maintaining long-term stabilization of the disease compared to au HSCT [55]. In our series, case 2 was treated with an HLA-haploidentical HSCT that led to clinical and radiological stability. This is the first case of a pediatric patient to benefit from such a treatment.

Proposed diagnostic and treatment algorithm for pediatric NMOSD is shown in Figure 3.

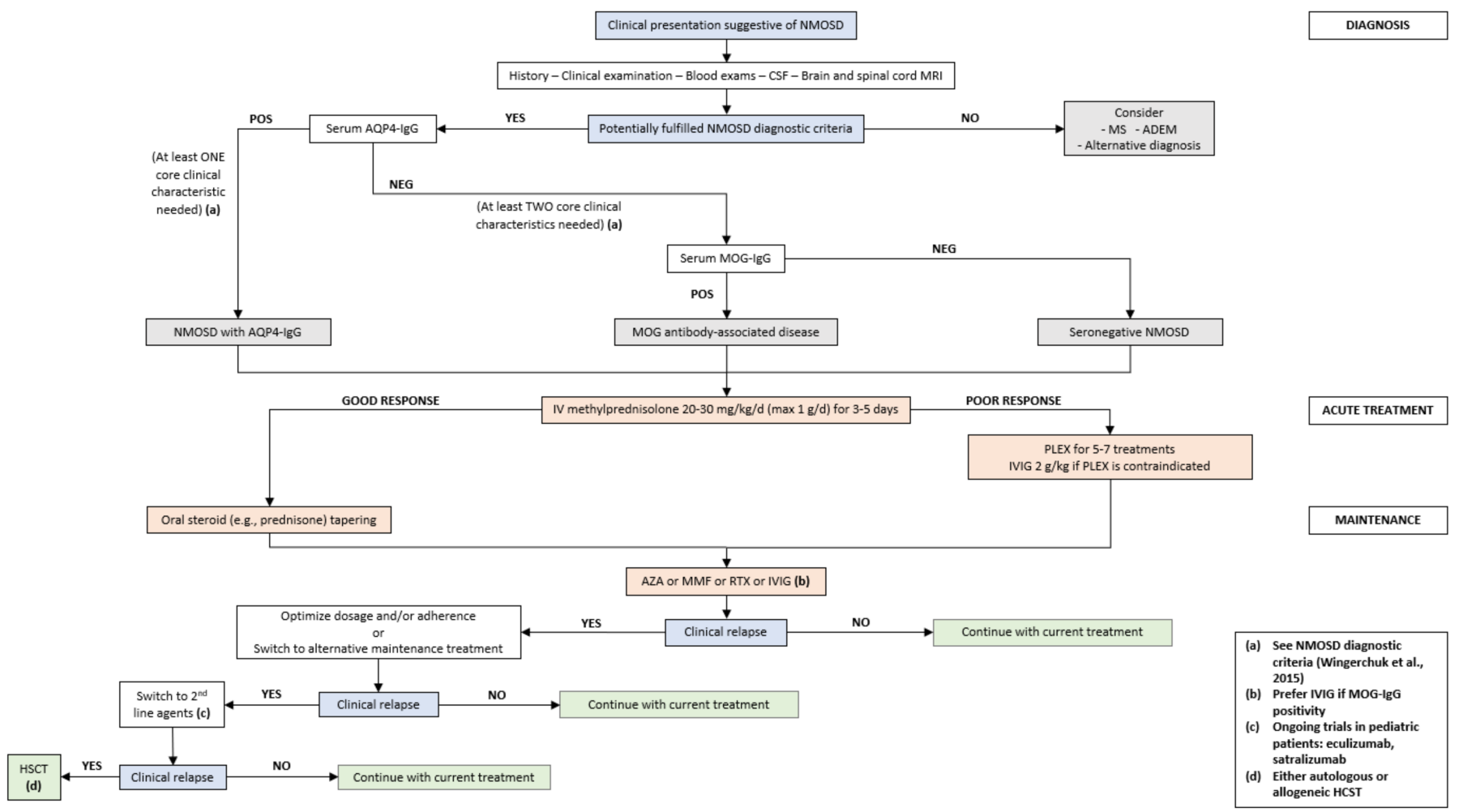

Figure 3. Proposed diagnostic and treatment algorithm for pediatric NMOSD. NMOSD: neuromyelitis optica spectrum disorders; CSF: cerebrospinal fluid; MRI: magnetic resonance imaging; MS: multiple sclerosis; ADEM: acute disseminated encephalomyelitis; AQP4: aquaporin-4; MOG: myelin oligodendrocyte glycoprotein; IV: intravenous; PLEX: plasma exchange; IVIG: intravenous immunoglobulin; AZA: azathioprine; MMF: mycophenolate mofetil; RTX: rituximab; HCST: hematopoietic stem cell transplantation.

\section{Conclusions}

Here, we have reviewed recent literature to provide a tool for diagnosing and treating children with NMOSD. These case reports are an example of the diagnostic and therapeutic complexity of pediatric NMOSD. Our cases, although limited in number, offer a wide range of personalized therapeutic strategies for the individual patient. Furthermore, our series also discusses AQP4-negative patients, unlike the other reviews that focus on AQP4positive pediatric patients.

In pediatric patients, diagnosis of NMOSD is often difficult, especially for patients who are seronegative for MOG and AQP4 and consequently the choice of therapy. Treatment options for pediatric patients with NMOSD are available and will continue to expand.

Thanks to new discoveries on the neurophysiological mechanisms underlying NMOSD, many new pharmacological agents are being studied, especially in the adult population.

However, further studies on long-term safety and treatment response of newer therapeutic agents are necessary, especially with regard to seronegative patients. 


\begin{abstract}
Author Contributions: Study design: M.A.N.F., M.V. and L.P.; data acquisition: M.A.N.F., R.P. and I.M.; data curation: M.A.N.F., C.R., G.S., R.M., F.U., S.T., M.P.C. and L.F.T.; writing original draft preparation: M.A.N.F., R.P., I.M.; writing review and editing: M.A.N.F., M.V. and L.P.; supervision: M.V. and F.V. All authors have read and agreed to the published version of the manuscript.
\end{abstract}

Funding: This research received no external funding.

Institutional Review Board Statement: We received Institutional Review Board Approval from Bambino Gesù Children's Hospital (date of approval: 29 July 2021).

Conflicts of Interest: The authors declare no conflict of interest.

\title{
References
}

1. Lennon, V.A.; Kryzer, T.J.; Pittock, S.J.; Verkman, A.S.; Hinson, S.R. IgG marker of optic-spinal multiple sclerosis binds to the aquaporin-4 water channel. J. Exp. Med. 2005, 202, 473-477. [CrossRef]

2. Saadoun, S.; Waters, P.; MacDonald, C.; Bell, B.A.; Vincent, A.; Verkman, A.S.; Papadopoulos, M.C. Neutrophil protease inhibition reduces neuromyelitis optica-immunoglobulin G-induced damage in mouse brain. Ann. Neurol. 2012, 71, 323-333. [CrossRef]

3. Zhang, H.; Verkman, A.S. Eosinophil pathogenicity mechanisms and therapeutics in neuromyelitis optica. J. Clin. Invest. 2013, 123, 2306-2316. [CrossRef] [PubMed]

4. Jasiak-Zatonska, M.; Kalinowska-Lyszczarz, A.; Michalak, S. The Immunology of Neuromyelitis Optica-Current Knowledge, Clinical Implications, Controversies and Future Perspectives. Int. J. Mol. Sci. 2016, 17, 273. [CrossRef] [PubMed]

5. Prasad, S.; Chen, J. What You Need to Know About AQP4, MOG, and NMOSD. Semin. Neurol. 2019, 39, 718-731. [CrossRef]

6. Rostasy, K.; Mader, S.; Schanda, K.; Peter, H.; Jutta Gärtner, J.; Kraus, V.; Karenfort, M.; Tibussek, D.; Blaschek, A.; Bajer-Kornek, B.; et al. Anti-myelin oligodendrocyte glycoprotein antibodies in pediatric patients with optic neuritis. Arch. Neurol. 2012, 69, 752-756. [CrossRef]

7. $\quad$ Ferilli, M.A.N.; Valeriani, M.; Papi, C.; Papetti, L.; Ruscitto, C.; Figà Talamanca, L.; Ursitti, F.; Moavero, R.; Vigevano, F.; Iorio, R. Clinical and neuroimaging characteristics of MOG autoimmunity in children with acquired demyelinating syndromes. Mult. Scler. Relat. Disord. 2021, 50, 102837. [CrossRef] [PubMed]

8. Absoud, M.; Lim, M.J.; Appleton, R.; Jacob, A.; Kitley, J.; Leite, M.I.; Pike, M.G.; Vincent, A.; Wassmer, E.; Waters, P.; et al. Paediatric neuromyelitis optica: Clinical, MRI of the brain and prognostic features. J. Neurol. Neurosurg. Psychiatry 2015, 86, 470-472. [CrossRef] [PubMed]

9. Quek, A.M.L.; McKeon, A.; Lennon, V.A.; Mandrekar, J.N.; Iorio, R.; Jiao, Y.; Costanzi, C.; Weinshenker, B.G.; Wingerchuk, D.M.; Lucchinetti, C.F.; et al. Effects of age and sex on aquaporin-4 autoimmunity. Arch. Neurol. 2012, 69, 1039-1043. [CrossRef] [PubMed]

10. McKeon, A.; Lennon, V.A.; Lotze, T.; Tenenbaum, S.; Ness, J.M.; Rensel, M.; Kuntz, N.L.; Fryer, J.P.; Homburger, H.; Hunter, J.; et al. CNS aquaporin-4 autoimmunity in children. Neurology 2008, 71, 93-100. [CrossRef] [PubMed]

11. Tenembaum, S.; Chitnis, T.; Nakashima, I.; Collongues, N.; McKeon, A.; Levy, M.; Rostasy, K. Neuromyelitis optica spectrum disorders in children and adolescents. Neurology 2016, 87, S59-S66. [CrossRef]

12. Palace, J.; Leite, M.I.; Nairne, A.; Vincent, A. Interferon Beta Treatment in Neuromyelitis Optica. Arch. Neurol. 2010, 67, 1016-1017. [CrossRef]

13. Jacob, A.; Hutchinson, M.; Elsone, L.; Kelly, S.; Ali, R.; Saukans, I.; Tubridy, N.; Boggild, M. Does natalizumab therapy worsen neuromyelitis optica? Neurology 2012, 79, 1065-1066. [CrossRef] [PubMed]

14. Min, J.-H.; Kim, B.J.; Lee, K.H. Development of extensive brain lesions following fingolimod (FTY720) treatment in a patient with neuromyelitis optica spectrum disorder. Mult. Scler. J. 2011, 18, 113-115. [CrossRef]

15. Wingerchuk, D.M.; Banwell, B.; Bennett, J.L.; Cabre, P.; Carroll, W.; Chitnis, T.; De Seze, J.; Fujihara, K.; Greenberg, B.; Jacob, A.; et al. International consensus diagnostic criteria for neuromyelitis optica spectrum disorders. Neurology 2015, 85, 177-189. [CrossRef]

16. Fragoso, Y.D.; Ferreira, M.L.; Oliveira, E.M.; Domingues, R.; Ribeiro, T.A.; Brooks, J.B.; Claudino, R.; Netto, J.M.; Gomes, S.; Adoni, T.; et al. Neuromyelitis Optica With Onset in Childhood and Adolescence. Pediatr. Neurol. 2014, 50, 66-68. [CrossRef]

17. Paolilo, R.B.; Hacohen, Y.; Yazbeck, E.; Armangue, T.; Bruijstens, A.; Lechner, C.; Apostolos-Pereira, S.L.; Martynenko, Y.; Breu, M.; Rimkus, C.D.M.; et al. Treatment and outcome of aquaporin-4 antibody-positive NMOSD. Neurol.—Neuroimmunol. Neuroinflamm. 2020, 7, e837. [CrossRef]

18. Chitnis, T.; Ness, J.; Krupp, L.; Waubant, E.; Hunt, T.; Olsen, C.S.; Rodriguez, M.; Lotze, T.; Gorman, M.; Benson, L.; et al. Clinical features of neuromyelitis optica in children. Neurology 2015, 86, 245-252. [CrossRef] [PubMed]

19. Khan, T.R.; Wang, C. Aquaporin-4 neuromyelitis optica spectrum disorder in a 2-year-old girl: Diagnostic and treatment considerations. Mult. Scler. Relat. Disord. 2020, 41, 102030. [CrossRef]

20. Gmuca, S.; Lieberman, S.; Mehta, J. Pediatric Neuromyelitis Optica Spectrum Disorder and Sjögren Syndrome: More Common Than Previously Thought? J. Rheumatol. 2017, 44, 959-960. [CrossRef] [PubMed]

21. Maraş, H.; Kara, B.; Anık, Y. Seropositive Neuromyelitis Optica: A Pediatric Case Report and 6-Year Follow-Up. Pediatr. Neurol. 2013, 49, 198-202. [CrossRef] 
22. Mahmood, N.A.; Silver, K.; Onel, K.; Ko, M.; Javed, A. Efficacy and Safety of Rituximab in Pediatric Neuromyelitis Optica. J. Child Neurol. 2010, 26, 244-247. [CrossRef]

23. Ikeda, A.; Watanabe, Y.; Kaba, H.; Kaneko, K.; Takahashi, T.; Takeshita, S. MRI findings in pediatric neuromyelitis optica spectrum disorder with MOG antibody: Four cases and review of the literature. Brain Dev. 2018, 41, 367-372. [CrossRef] [PubMed]

24. Chuquilin, M.; Mullaguri, N.; Weinshenker, B. Pediatric familial neuromyelitis optica in two sisters with long term follow-up. J. Clin. Neurosci. 2016, 29, 183-184. [CrossRef]

25. Lotze, T.E.; Northrop, J.L.; Hutton, G.J.; Ross, B.; Schiffman, J.S.; Hunter, J.V. Spectrum of Pediatric Neuromyelitis Optica. Pediatrics 2008, 122, e1039-e1047. [CrossRef] [PubMed]

26. Rostásy, K.; Mader, S.; Hennes, E.M.; Schanda, K.; Gredler, V.; Guenther, A.; Blaschek, A.; Korenke, C.; Pritsch, M.; Pohl, D.; et al. Persisting myelin oligodendrocyte glycoprotein antibodies in aquaporin-4 antibody negative pediatric neuromyelitis optica. Mult. Scler. J. 2012, 19, 1052-1059. [CrossRef]

27. Dimitrijevic, N.; Bogicevic, D.; Dimitrijevic, A.; Nikolic, D. Early presentation of neuromyelitis optica. Indian Pediatr. 2012, 49, 924-925. [CrossRef] [PubMed]

28. Loma, I.P.; Asato, M.R.; Filipink, R.A.; Alper, G. Neuromyelitis Optica in a Young Child with Positive Serum Autoantibody. Pediatr. Neurol. 2008, 39, 209-212. [CrossRef]

29. Yavuz, H.; Kireşi, D. Unusual Manifestations of Pediatric Neuromyelitis Optica. J. Child Neurol. 2012, 28, 658-662. [CrossRef] [PubMed]

30. Marino, A.; Narula, S.; Lerman, M.A. First Pediatric Patient With Neuromyelitis Optica and Sjögren Syndrome Successfully Treated With Tocilizumab. Pediatr. Neurol. 2017, 73, e5-e6. [CrossRef]

31. He, D.; Yu, Y.; Yan, W.; Dai, Q.; Xu, Z.; Chu, L. Individualized Rituximab Treatment for Relapsing Neuromyelitis Optica: A Pediatric Case Report. Pediatr. Neurol. 2014, 51, 255-258. [CrossRef]

32. Elpers, C.; Gross, C.; Fiedler, B.; Meuth, S.G.; Kurlemann, G. A Case Report on Juvenile Neuromyelitis Optica: Early Onset, Long Remission Period, and Atypical Treatment Response. Neuropediatrics 2015, 46, 292-295. [CrossRef]

33. Maillart, E.; Renaldo, F.; Papeix, C.; Deiva, K.; Bonheur, J.; Kwon, T.; Boespflug-Tanguy, O.; Germanaud, D.; Marignier, R. Dramatic efficacy of ofatumumab in refractory pediatric-onset AQP4-IgG neuromyelitis optica spectrum disorder. Neurol.-Neuroimmunol. Neuroinflamm. 2020, 7, e683. [CrossRef] [PubMed]

34. Kamawal, A.; Hoerning, S.; Galiano, M.; Rompel, O.; Trollmann, R. Myelin Oligodendrocyte Glycoprotein Antibody-Associated Neuromyelitis Optica Spectrum Disorder in a 6-Year-Old Boy. Klin. Pädiatrie 2019, 231, 220-223. [CrossRef]

35. Hudson, L.A.; Bernard, T.J.; Tseng, B.S.; Miller, B.R.; Corboy, J.R. Neuromyelitis Optica Immunoglobulin G in a Child. Pediatr. Neurol. 2006, 35, 370-372. [CrossRef]

36. Gokce, G.; Ceylan, O.M.; Mutlu, F.M.; I Altinsoy, H.; Koylu, M.T. Relapsing Devic's disease in a child. J. Pediatr. Neurosci. 2013, 8 , 146-149. [CrossRef] [PubMed]

37. Khan, T.R.; Zimmern, V.; Aquino, V.; Wang, C. Autologous hematopoietic stem cell transplantation in a pediatric patient with aquaporin-4 neuromyelitis optica spectrum disorder. Mult. Scler. Relat. Disord. 2021, 50, 102852. [CrossRef]

38. Arnold, T.W.; Myers, G.J. Neuromyelitis optica (Devic syndrome) in a 12-year-old male with complete recovery following steroids. Pediatr. Neurol. 1987, 3, 313-315. [CrossRef]

39. Davis, R.; Thiele, E.; Barnes, P.; Riviello, J.J. Neuromyelitis Optica in Childhood: Case Report With Sequential MRI Findings. J. Child Neurol. 1996, 11, 164-167. [CrossRef] [PubMed]

40. Peña, J.A.; Ravelo, M.E.; Cruz, E.M.-L.; Montiel-Nava, C. NMO in pediatric patients: Brain involvement and clinical expression. Arq. De Neuro-Psiquiatr. 2011, 69, 34-38. [CrossRef]

41. Numata, Y.; Uematsu, M.; Suzuki, S.; Miyabayashi, T.; Oyama, T.; Kubota, S.; Itoh, T.; Hino-Fukuyo, N.; Takahashi, T.; Kure, S. Aquaporin-4 autoimmunity in a child without optic neuritis and myelitis. Brain Dev. 2015, 37, 149-152. [CrossRef] [PubMed]

42. Milani, N.; Zibordi, F.; Erbetta, A.; Pollo, B.; Angelini, L. Neuromyelitis Optica in a Child with Atypical Onset and Severe Outcome. Neuropediatrics 2004, 35, 198-201. [CrossRef]

43. Longoni, G.; Bigi, S.; Branson, H.M.; Hawkins, C.; Rutka, J.T.; Filippi, M.; Yeh, E.A. Multicystic demyelinating myelopathy: Widening spectrum of pediatric aquaporin-4 autoimmunity. Neurology 2014, 82, 902-903. [CrossRef]

44. Bianchi, A.; Bartolini, E.; Melani, F.; Guerrini, R.; Mascalchi, M. Isolated recurrent myelitis in a 7-year-old child with serum aquaporin-4 IgG antibodies. J. Neurol. 2016, 264, 179-181. [CrossRef]

45. Arabshahi, B. Devic Disease in a Child with Primary Sjögren Syndrome. J. Child Neurol. 2006, 21, 284-286. [CrossRef]

46. Zhang, Z.; Zhou, H.; Liu, X.; Liu, L.; Shu, S.; Fang, F. Identification of the clinical and neuroimaging characteristics in children with neuromyelitis optica spectrum disorders: A case series. Transl. Pediatr. 2021, 10, 2459-2466. [CrossRef]

47. Baghbanian, S.M.; Sahraian, M.A.; Moghadasi, A.N.; Asgari, N. Disability and Therapeutic Response in Paediatric Neuromyelitis Optica Spectrum Disorder: A Case Series from Iran. Iran. J. Child Neurol. 2019, 13, 99-104. [PubMed]

48. Dembinski, K.; Gieron-Korthals, M.; Martinez, C.R.; Rodriguez, L. Neuromyelitis Optica in Child: Diagnostic and Therapeutic Challenges. Case Rep. Pediatr. 2013, 2013, 1-4. [CrossRef]

49. Farhat, L.; Dara, J.; Duberstein, S.; De, A. Secondary Hypogammaglobulinemia After Rituximab for Neuromyelitis Optica: A Case Report. Drug Saf.-Case Rep. 2018, 5, 22. [CrossRef] [PubMed] 
50. Nosadini, M.; Alper, G.; Riney, C.J.; Benson, L.A.; Mohammad, S.S.; Ramanathan, S.; Nolan, M.; Appleton, R.; Leventer, R.J.; Deiva, K.; et al. Rituximab monitoring and redosing in pediatric neuromyelitis optica spectrum disorder. Neurol.-Neuroimmunol. Neuroinflamm. 2016, 3, e188. [CrossRef]

51. Lechner, C.; Baumann, M.; Hennes, E.-M.; Schanda, K.; Marquard, K.; Karenfort, M.; Leiz, S.; Pohl, D.; Venkateswaran, S.; Pritsch, M.; et al. Antibodies to MOG and AQP4 in children with neuromyelitis optica and limited forms of the disease. J. Neurol. Neurosurg. Psychiatry 2015, 87, 897-905. [CrossRef]

52. Longoni, G.; Banwell, B.; Filippi, M.; Yeh, E.A. Rituximab as a first-line preventive treatment in pediatric NMOSDs. Neurol.Neuroimmunol. Neuroinflamm. 2014, 1, e46. [CrossRef]

53. Camera, V.; Messina, S.; Elhadd, K.T.; Sanpera-Iglesias, J.; Mariano, R.; Hacohen, Y.; Dobson, R.; Meletti, S.; Wassmer, E.; Lim, M.J.; et al. Early predictors of disability of paediatric-onset AQP4-IgG-seropositive neuromyelitis optica spectrum disorders. J. Neurol. Neurosurg. Psychiatry 2021, 93, 101-111. [CrossRef]

54. Bradshaw, M.J.; Vu, N.; Hunley, T.E.; Chitnis, T. Child Neurology: Neuromyelitis optica spectrum disorders. Neurology 2017, 88, e10-e13. [CrossRef]

55. Ceglie, G.; Papetti, L.; Valeriani, M.; Merli, P. Hematopoietic Stem Cell Transplantation in Neuromyelitis Optica-Spectrum Disorders (NMO-SD): State-of-the-Art and Future Perspectives. Int. J. Mol. Sci. 2020, 21, 5304. [CrossRef] [PubMed]

56. Tillema, J.; McKeon, A. The Spectrum of Neuromyelitis Optica (NMO) in Childhood. J. Child Neurol. 2012, 27, 1437-1447. [CrossRef] [PubMed]

57. Tenembaum, S.; Yeh, E.A.; Alroughani, R.; Altintas, A.; Amezcua, L.; Apiwattanakul, M.; Asgari, N.; Banwell, B.; Abboud, H.; The Guthy-Jackson Foundation International Clinical Consortium (GJCF-ICC); et al. Pediatric NMOSD: A Review and Position Statement on Approach to Work-Up and Diagnosis. Front. Pediatr. 2020, 8, 339. [CrossRef]

58. Lennon, V.A.; Wingerchuk, D.M.; Kryzer, T.J.; Pittock, S.J.; Lucchinetti, C.F.; Fujihara, K.; Nakashima, I.; Weinshenker, B.G. A serum autoantibody marker of neuromyelitis optica: Distinction from multiple sclerosis. Lancet 2004, 364, 2106-2112. [CrossRef]

59. Wingerchuk, D.M.; Lennon, V.A.; Pittock, S.J.; Lucchinetti, C.F.; Weinshenker, B.G. Revised diagnostic criteria for neuromyelitis optica. Neurology 2006, 66, 1485-1489. [CrossRef] [PubMed]

60. Tenembaum, S.; Chitnis, T.; Ness, J.; Hahn, J.S. Acute disseminated encephalomyelitis. Neurology 2007, 68, S23-S36. [CrossRef] [PubMed]

61. Rosenthal, J.F.; Hoffman, B.M.; Tyor, W.R. CNS inflammatory demyelinating disorders: MS, NMOSD and MOG antibody associated disease. J. Investig. Med. 2019, 68, 321-330. [CrossRef]

62. Baghbanian, S.M.; Asgari, N.; Sahraian, M.A.; Moghadasi, A.N. A comparison of pediatric and adult neuromyelitis optica spectrum disorders: A review of clinical manifestation, diagnosis, and treatment. J. Neurol. Sci. 2018, 388, 222-231. [CrossRef] [PubMed]

63. Huda, S.; Whittam, D.; Bhojak, M.; Chamberlain, J.; Noonan, C.; Jacob, A.; Kneen, R. Neuromyelitis optica spectrum disorders Clin. Med. 2019, 19, 169-176. [CrossRef] [PubMed]

64. Shosha, E.; Dubey, D.; Palace, J.; Nakashima, I.; Jacob, A.; Fujihara, K.; Takahashi, T.; Whittam, D.; Leite, M.I.; Misu, T.; et al. Area postrema syndrome. Neurology 2018, 91, e1642-e1651. [CrossRef] [PubMed]

65. Zantah, M.; Coyle, T.B.; Datta, D. Acute Respiratory Failure due to Neuromyelitis Optica Treated Successfully with Plasmapheresis. Case Rep. Pulmonol. 2016, 2016, 1-3. [CrossRef] [PubMed]

66. Iorio, R.; Lucchinetti, C.F.; Lennon, V.A.; Costanzi, C.; Hinson, S.; Weinshenker, B.G.; Pittock, S.J. Syndrome of inappropriate antidiuresis may herald or accompany neuromyelitis optica. Neurology 2011, 77, 1644-1646. [CrossRef]

67. Salama, S.; Khan, M.; Pardo, S.; Izbudak, I.; Levy, M. MOG antibody-associated encephalomyelitis/encephalitis. Mult. Scler. 2019, 25, 1427-1433. [CrossRef]

68. Shahmohammadi, S.; Doosti, R.; Shahmohammadi, A.; Mohamadianinejad, S.E.; Sahraian, M.A.; Azimi, A.R.; Harirchian, M.H.; Asgari, N.; Moghadasi, A.N. Autoimmune diseases associated with Neuromyelitis Optica Spectrum Disorders: A literature review. Mult. Scler. Relat. Disord. 2018, 27, 350-363. [CrossRef]

69. Kornitzer, J.M.; Kimura, Y.; Janow, G.L. Primary Sjögren Syndrome in a Child with a Neuromyelitis Optica Spectrum Disorder. J. Rheumatol. 2016, 43, 1260-1261. [CrossRef]

70. Sarigecili, E.; Cobanogullari, M.D.; Komur, M.; Okuyaz, C. A rare concurrence: Antibodies against Myelin Oligodendrocyte Glycoprotein and N-methyl-d-aspartate receptor in a child. Mult. Scler. Relat. Disord. 2018, 28, 101-103. [CrossRef]

71. Tao, S.; Zhang, Y.; Ye, H.; Guo, D. AQP4-IgG-seropositive neuromyelitis optica spectrum disorder (NMOSD) coexisting with anti-N-methyl-D-aspartate receptor (NMDAR) encephalitis: A case report and literature review. Mult. Scler. Relat. Disord. 2019, 35, 185-192. [CrossRef]

72. Mealy, M.A.; Whetstone, A.; Orman, G.; Izbudak, I.; Calabresi, P.; Levy, M. Longitudinally extensive optic neuritis as an MRI biomarker distinguishes neuromyelitis optica from multiple sclerosis. J. Neurol. Sci. 2015, 355, 59-63. [CrossRef] [PubMed]

73. Kupersmith, M.J.; Alban, T.; Zeiffer, B.; Lefton, D. Contrast-enhanced MRI in acute optic neuritis: Relationship to visual performance. Brain 2002, 125, 812-822. [CrossRef]

74. Asgari, N.; Skejoe, H.P.B.; Lillevang, S.T.; Steenstrup, T.; Stenager, E.; Kyvik, K.O. Modifications of longitudinally extensive transverse myelitis and brainstem lesions in the course of neuromyelitis optica (NMO): A population-based, descriptive study. BMC Neurol. 2013, 13, 1-8. [CrossRef] 
75. Kim, H.J.; Paul, F.; Lana-Peixoto, M.A.; Tenembaum, S.; Asgari, N.; Palace, J.; Klawiter, E.C.; Sato, D.K.; de Seze, J.; Wuerfel, J.; et al. MRI characteristics of neuromyelitis optica spectrum disorder: An international update. Neurology 2015, 84, 1165-1173. [CrossRef] [PubMed]

76. Banwell, B.; Tenembaum, S.; Lennon, V.A.; Ursell, E.; Kennedy, J.; Bar-Or, A.; Weinshenker, B.G.; Lucchinetti, C.F.; Pittock, S.J. Neuromyelitis optica-IgG in childhood inflammatory demyelinating CNS disorders. Neurology 2007, 70, 344-352. [CrossRef] [PubMed]

77. Banwell, B.; Arnold, D.L.; Tillema, J.-M.; Rocca, M.A.; Filippi, M.; Weinstock-Guttman, B.; Zivadinov, R.; Sormani, M.P. MRI in the evaluation of pediatric multiple sclerosis. Neurology 2016, 87, S88-S96. [CrossRef]

78. Pittock, S.J.; Lennon, V.A.; Krecke, K.; Wingerchuk, D.M.; Lucchinetti, C.F.; Weinshenker, B.G. Brain Abnormalities in Neuromyelitis Optica. Arch. Neurol. 2006, 63, 390-396. [CrossRef] [PubMed]

79. Kim, W.; Park, M.S.; Lee, S.H.; Kim, S.-H.; Jung, I.J.; Takahashi, T.; Misu, T.; Fujihara, K.; Kim, H.J. Characteristic brain magnetic resonance imaging abnormalities in central nervous system aquaporin-4 autoimmunity. Mult. Scler. J. 2010, 16, 1229-1236. [CrossRef]

80. Majed, M.; Fryer, J.P.; McKeon, A.; Lennon, V.A.; Pittock, S.J. Clinical utility of testing AQP4-IgG in CSF. Neurol.-Neuroimmunol. Neuroinflamm. 2016, 3, e231. [CrossRef]

81. Jiao, Y.; Fryer, J.P.; Lennon, V.A.; Jenkins, S.M.; Quek, A.M.L.; Smith, C.Y.; McKeon, A.; Costanzi, C.; Iorio, R.; Weinshenker, B.G.; et al. Updated estimate of AQP4-IgG serostatus and disability outcome in neuromyelitis optica. Neurology 2013, 81, 1197-1204. [CrossRef] [PubMed]

82. Kitley, J.; Woodhall, M.; Waters, P.; Leite, M.I.; Devenney, E.; Craig, J.; Palace, J.; Vincent, A. Myelin-oligodendrocyte glycoprotein antibodies in adults with a neuromyelitis optica phenotype. Neurology 2012, 79, 1273-1277. [CrossRef] [PubMed]

83. Jarius, S.; Ruprecht, K.; Kleiter, I.; Borisow, N.; Asgari, N.; Pitarokoili, K.; Pache, F.; Stich, O.; Beume, L.-A.; Hümmert, M.W.; et al. MOG-IgG in NMO and related disorders: A multicenter study of 50 patients. Part 2: Epidemiology, clinical presentation, radiological and laboratory features, treatment responses, and long-term outcome. J. Neuroinflamm. 2016, 13, 1-45. [CrossRef]

84. Waters, P.; Woodhall, M.; O'Connor, K.C.; Reindl, M.; Lang, B.; Sato, D.K.; Juryńczyk, M.; Tackley, G.; Rocha, J.; Takahashi, T.; et al. MOG cell-based assay detects non-MS patients with inflammatory neurologic disease. Neurol.-Neuroimmunol. Neuroinflamm. 2015, 2, e89. [CrossRef]

85. Bergamaschi, R.; Tonietti, S.; Franciotta, D.; Candeloro, E.; Tavazzi, E.; Piccolo, G.; Romani, A.; Cosi, V. Oligoclonal bands in Devic's neuromyelitis optica and multiple sclerosis: Differences in repeated cerebrospinal fluid examinations. Mult. Scler. J. 2004, 10, 2-4. [CrossRef]

86. Jarius, S.; Paul, F.; Franciotta, D.; Ruprecht, K.; Ringelstein, M.; Bergamaschi, R.; Rommer, P.; Kleiter, I.; Stich, O.; Reuss, R.; et al. Cerebrospinal fluid findings in aquaporin-4 antibody positive neuromyelitis optica: Results from 211 lumbar punctures. J. Neurol. Sci. 2011, 306, 82-90. [CrossRef]

87. Romeo, A.R.; Segal, B. Treatment of neuromyelitis optica spectrum disorders. Curr. Opin. Rheumatol. 2019, 31, 250-255. [CrossRef]

88. Palace, J.; Leite, I.; Jacob, A. A practical guide to the treatment of neuromyelitis optica. Pract. Neurol. 2012, 12, 209-214. [CrossRef] [PubMed]

89. Sellner, J.; Boggild, M.; Clanet, M.; Hintzen, R.Q.; Illes, Z.; Montalban, X.; Du Pasquier, R.; Polman, C.H.; Sorensen, P.S.; Hemmer B. EFNS guidelines on diagnosis and management of neuromyelitis optica. Eur. J. Neurol. 2010, 17, 1019-1032. [CrossRef]

90. Weinshenker, B.G.; O’Brien, P.C.; Petterson, T.M.; Noseworthy, J.H.; Lucchinetti, C.F.; Dodick, D.W.; Pineda, A.A.; Stevens, L.N.; Rodriguez, M. A randomized trial of plasma exchange in acute central nervous system inflammatory demyelinating disease. Ann. Neurol. 1999, 46, 878-886. [CrossRef]

91. Abboud, H.; Petrak, A.; Mealy, M.; Sasidharan, S.; Siddique, L.; Levy, M. Treatment of acute relapses in neuromyelitis optica: Steroids alone versus steroids plus plasma exchange. Mult. Scler. J. 2015, 22, 185-192. [CrossRef]

92. Elsone, L.; Panicker, J.; Mutch, K.; Boggild, M.; Appleton, R.; Jacob, A. Role of intravenous immunoglobulin in the treatment of acute relapses of neuromyelitis optica: Experience in 10 patients. Mult. Scler. J. 2013, 20, 501-504. [CrossRef]

93. Trebst, C.; Jarius, S.; Berthele, A.; Paul, F.; Schippling, S.; Wildemann, B.; Borisow, N.; Kleiter, I.; Aktas, O.; Kümpfel, T.; et al. Update on the diagnosis and treatment of neuromyelitis optica: Recommendations of the Neuromyelitis Optica Study Group (NEMOS). J. Neurol. 2013, 261, 1-16. [CrossRef] [PubMed]

94. Hacohen, Y.; Banwell, B. Treatment Approaches for MOG-Ab-Associated Demyelination in Children. Curr. Treat. Opt. Neurol. 2019, 21, 1-14. [CrossRef]

95. Kitley, J.; Leite, M.I.; Nakashima, I.; Waters, P.; McNeillis, B.; Brown, R.; Takai, Y.; Takahashi, T.; Misu, T.; Elsone, L.; et al. Prognostic factors and disease course in aquaporin-4 antibody-positive patients with neuromyelitis optica spectrum disorder from the United Kingdom and Japan. Brain 2012, 135, 1834-1849. [CrossRef]

96. Montcuquet, A.; Collongues, N.; Papeix, C.; Zephir, H.; Audoin, B.; Laplaud, D.; Bourre, B.; Brochet, B.; Camdessanche, J.-P.; Labauge, P.; et al. Effectiveness of mycophenolate mofetil as first-line therapy in AQP4-IgG, MOG-IgG, and seronegative neuromyelitis optica spectrum disorders. Mult. Scler. J. 2016, 23, 1377-1384. [CrossRef] [PubMed]

97. Wingerchuk, D.M. Neuromyelitis Optica: Potential Roles for Intravenous Immunoglobulin. J. Clin. Immunol. 2012, 33, 33-37. [CrossRef]

98. Viswanathan, S.; Wong, A.H.; Quek, A.M.; Yuki, N. Intravenous immunoglobulin may reduce relapse frequency in neuromyelitis optica. J. Neuroimmunol. 2015, 282, 92-96. [CrossRef] 
99. Magraner, M.; Coret, F.; Casanova, B. The effect of intravenous immunoglobulin on neuromyelitis optica. Neurologia 2013, 28, 65-72. [CrossRef]

100. Costanzi, C.; Matiello, M.; Lucchinetti, C.F.; Weinshenker, B.G.; Pittock, S.J.; Mandrekar, J.; Thapa, P.; McKeon, A. Azathioprine: Tolerability, efficacy, and predictors of benefit in neuromyelitis optica. Neurology 2011, 77, 659-666. [CrossRef] [PubMed]

101. Elsone, L.; Kitley, J.; Luppe, S.; Lythgoe, D.; Mutch, K.; Jacob, S.; Brown, R.; Moss, K.; McNeillis, B.; Goh, Y.Y.; et al. Long-term efficacy, tolerability and retention rate of azathioprine in 103 aquaporin- 4 antibody-positive neuromyelitis optica spectrum disorder patients: A multicentre retrospective observational study from the UK. Mult. Scler. J. 2014, 20, 1533-1540. [CrossRef] [PubMed]

102. Goldsmith, D.; Carrey, E.A.; Edbury, S.; Smolenski, R.; Jagodzinski, P.; Simmonds, H.A. Mycophenolate mofetil, an inhibitor of inosine monophosphate dehydrogenase, causes a paradoxical elevation of GTP in erythrocytes of renal transplant patients. Clin. Sci. 2004, 107, 63-68. [CrossRef] [PubMed]

103. Huh, S.-Y.; Kim, S.-H.; Hyun, J.-W.; Joung, A.-R.; Park, M.S.; Kim, B.-J.; Kim, H.J. Mycophenolate Mofetil in the Treatment of Neuromyelitis Optica Spectrum Disorder. JAMA Neurol. 2014, 71, 1372-1378. [CrossRef]

104. Jacob, A.; Matiello, M.; Weinshenker, B.G.; Wingerchuk, D.M.; Lucchinetti, C.; Shuster, E.; Carter, J.; Keegan, B.M.; Kantarci, O.H.; Pittock, S.J. Treatment of Neuromyelitis Optica With Mycophenolate Mofetil. Arch. Neurol. 2009, 66, 1128-1133. [CrossRef]

105. Nosadini, M.; Gadian, J.; Lim, M.; Sartori, S.; Thomas, T.; Dale, R.C. Mycophenolate mofetil in paediatric autoimmune or immune-mediated diseases of the central nervous system: Clinical experience and recommendations. Dev. Med. Child Neurol. 2018, 61, 458-468. [CrossRef]

106. Dale, R.C.; Brilot, F.; Duffy, L.V.; Twilt, M.; Waldman, A.T.; Narula, S.; Muscal, E.; Deiva, K.; Andersen, E.; Eyre, M.R.; et al Utility and safety of rituximab in pediatric autoimmune and inflammatory CNS disease. Neurology 2014, 83, 142-150. [CrossRef] [PubMed]

107. Kimbrough, D.; Fujihara, K.; Jacob, A.; Lana-Peixoto, M.A.; Leite, M.I.; Levy, M.; Marignier, R.; Nakashima, I.; Palace, J.; de Seze, J.; et al. Treatment of neuromyelitis optica: Review and recommendations. Mult. Scler. Relat. Disord. 2012, 1, 180-187. [CrossRef]

108. Gürcan, H.M.; Keskin, D.B.; Stern, J.N.; Nitzberg, M.A.; Shekhani, H.; Ahmed, A.R. A review of the current use of rituximab in autoimmune diseases. Int. Immunopharmacol. 2009, 9, 10-25. [CrossRef]

109. Marcinnò, A.; Marnetto, F.; Valentino, P.; Martire, S.; Balbo, A.; Drago, A.; Leto, M.; Capobianco, M.; Panzica, G.; Bertolotto, A. Rituximab-induced hypogammaglobulinemia in patients with neuromyelitis optica spectrum disorders. Neurol.-Neuroimmunol. Neuroinflamm. 2018, 5, e498. [CrossRef]

110. Hacohen, Y.; Wong, Y.Y.; Lechner, C.; Jurynczyk, M.; Wright, S.; Konuskan, B.; Kalser, J.; Poulat, A.L.; Maurey, H.; GanelinCohen, E.; et al. Disease Course and Treatment Responses in Children With Relapsing Myelin Oligodendrocyte Glycoprotein Antibody-Associated Disease. JAMA Neurol. 2018, 75, 478-487. [CrossRef]

111. Pittock, S.J.; Berthele, A.; Fujihara, K.; Kim, H.J.; Levy, M.; Palace, J.; Nakashima, I.; Terzi, M.; Totolyan, N.; Viswanathan, S.; et al Eculizumab in Aquaporin-4-Positive Neuromyelitis Optica Spectrum Disorder. N. Engl. J. Med. 2019, 381, 614-625. [CrossRef]

112. McNamara, L.A.; Topaz, N.; Wang, X.; Hariri, S.; Fox, L.; MacNeil, J.R. High Risk for Invasive Meningococcal Disease Among Patients Receiving Eculizumab (Soliris) Despite Receipt of Meningococcal Vaccine. MMWR. Morb. Mortal. Wkly. Rep. 2017, 66, 734-737. [CrossRef] [PubMed]

113. Alexion Pharmaceuticals. A Phase 2/3 Open-Label, Single-Arm Trial to Evaluate the Safety and Activity of Eculizumab in Pediatric Patients with Relapsing Neuromyelitis Optica Spectrum Disorder. Available online: https://clinicaltrials.gov/ct2 / show / NCT04155424 (accessed on 27 June 2021).

114. Araki, M.; Matsuoka, T.; Miyamoto, K.; Kusunoki, S.; Okamoto, T.; Murata, M.; Miyake, S.; Aranami, T.; Yamamura, T. Efficacy of the anti-IL-6 receptor antibody tocilizumab in neuromyelitis optica: A pilot study. Neurology 2014, 82, 1302-1306. [CrossRef] [PubMed]

115. Yamamura, T.; Kleiter, I.; Fujihara, K.; Palace, J.; Greenberg, B.; Zakrzewska-Pniewska, B.; Patti, F.; Tsai, C.-P.; Saiz, A.; Yamazaki, H.; et al. Trial of Satralizumab in Neuromyelitis Optica Spectrum Disorder. N. Engl. J. Med. 2019, 381, 2114-2124. [CrossRef] [PubMed]

116. Hoffmann-La Roche. A Multicenter, Single Arm, Open-Label Study to Evaluate the Long-Term Safety and Efficacy of Satralizumab in Patients with Neuromyelitis Optica Spectrum Disorder (NMOSD). Available online: https://clinicaltrials.gov/ct2/show/ NCT04660539 (accessed on 22 July 2021).

117. Frampton, J.E. Inebilizumab: First Approval. Drugs 2020, 80, 1259-1264. [CrossRef] [PubMed]

118. Cree, B.A.C.; Bennett, J.L.; Kim, H.J.; Weinshenker, B.G.; Pittock, S.J.; Wingerchuk, D.M.; Fujihara, K.; Paul, F.; Cutter, G.R.; Marignier, R.; et al. Inebilizumab for the treatment of neuromyelitis optica spectrum disorder (N-MOmentum): A double-blind, randomised placebo-controlled phase $2 / 3$ trial. Lancet 2019, 394, 1352-1363. [CrossRef]

119. Greco, R.; Bondanza, A.; Oliveira, M.C.; Badoglio, M.; Burman, J.; Piehl, F.; Hagglund, H.; Krasulová, E.; Simoes, B.; Carlson, K.; et al. Autologous hematopoietic stem cell transplantation in neuromyelitis optica: A registry study of the EBMT Autoimmune Diseases Working Party. Mult. Scler. J. 2014, 21, 189-197. [CrossRef] 